\section{Klinische Pharmakologie und therapeutische Anwendung steroidfreier Entzündungshemmer beim Pferd}

\author{
P. Lees und A. J. Higgins \\ Department of Physiology, Royal Veterinary College, \\ North Mymms, Hatfield
}

\section{Einführung}

Die klinische Pharmakologie beinhaltet eine Untersuchung aller (erwünschten und auch unerwünschten) Wirkungen eines Arzneimittelstoffs und seinen Metabolismus in einer einzelnen Art von lebendem Organismus. Dazu gehört eine Erforschung der Absorptionsweise des Medikaments im Körper.

Die steroidfreien Entzündungshemmer (NSAIDs) finden sich an manchen Stellen als Substanzen definiert, die, anders als Steroide, einen oder mehrere Schritte der entzündlichen Reaktion unterdrücken. Gewöhnlich dient der Begriff jedoch in einem enger umschriebenen Bereich zur Bezeichnung antiphlogistischer pharmakologischer Wirkstof$\mathrm{fe}$, nämlich nur solcher, die klinisch zur Anwendung kommen, und, noch spezieller, solcher, deren Wirkung in der Hemmung eines Teils eines Enzymsystems besteht; dieses System wandelt Arachidonsäure in einige höchst wirksame Entzündungsmediatoren um (Higgins und Lees, 1984).

NSAIDs sind schwache organische Säuren mit ähnlichen antipyretischen, analgetischen und auch entzündungshemmenden Effekten wie Aspirin. Vogel (1982) schrieb: „NSAIDs haben zwar einen großen Fortschritt in der Pferdepraxis eingeleitet und das Arbeitsleben Tausender Pferde verlängert, ihre Entdeckung hat aber auch ihre Probleme mit sich gebracht." Dieser Überblick befaßt sich mit den Wirkungsformen, Stoffwechselwegen und Anwendungsmöglichkeiten der oben genannten Verbindungen beim Pferd. Andere steroidfreie Wirkstoffe, die in der Humanmedizin oder, weniger verbreitet, in der Veterinärmedizin klinisch in Gebrauch sind, darunter Goldsalze, Penizillamin, Orgotein und Hyaluronsäure, klammern wir aus. Ihre Wirkungsweise unterscheidet sich doch vermutlich von der des Aspirins, Phenylbutazons und verwandter Verbindungen.

Speziesspezifische Abweichungen im Hinblick auf die Art der Wirkung sind sehr wohl berücksichtigt. Außerdem kam in den vergangenen Jahren die Möglichkeit rassebedingter Unterschiede beim Pferd, die Absorption, Ausscheidung und Empfindlichkeit gegenüber den toxischen Effekten der NSAIDs betreffend, zur Sprache. Sie haben

\begin{abstract}
Zusammenfassung
Schwache organische Säuren mit entzündungshemmenden, analgetischen und antipyretischen Eigenschaften - gemeinhin unter dem Namen aspirinartige Medikamente bekannt - sind in der Pferdeheilkunde seit fast 100 Jahren in Gebrauch. Diese steroidfreien Antiphlogistika (NSAIDs = non-steroidal anti-inflammatory drugs) lassen sich chemisch in zwei Gruppen einteilen; die Enolsäuren mit Phenylbutazon und die Carbonsäuren wie Flunixin, Meclofenamat und Naproxen. Alle NSAIDs haben eine ähnliche, möglicherweise auch dieselbe Wirkungsweise. Sie ist sowohl für die therapeutischen als auch für die toxischen Effekte der NSAIDs verantwortlich. Sie blockieren teilweise den Zyklooxygenase-Enzymstoffwechsel und unterdrücken dadurch die Synthese verschiedener chemischer Entzündungsmediatoren, gewöhnlich als Eikosane bekannt. Den bisherigen Erkenntnissen nach zu urteilen, besitzen einige neuere NSAIDs bei der Dosierung einen ausreichenden Sicherheitsspielraum, aber es bedarf weiterer Forschungsarbeit. Die Toxizität von Phenylbutazon beim Pferd wurde in den vergangenen Jahren besonders eingehenden Untersuchungen unterzogen. Es hat sich dabei als nephrotoxisch und, was von größter Bedeutung war, als Verursacher von Ulcera im Magen-Darm-Trakt erwiesen, wenn die verabreichten Dosen relativ hoch waren. Wahrscheinlich wirken unterschiedliche Faktoren prädisponierend bei der Phenylbutazon-Toxizität, darunter Rasse und Alter, aber der hohen Dosierung kommt, so glaubt man, besondere Bedeutung zu. Unsere Betrachtungen richten sich auf die Absorption und den weiteren Verbleib der NSAIDs im Organismus. Besondere Aufmerksamkeit erfahren auch die Stoffwechselwege, auf welchen diese pharmakokinetischen Eigenschaften zur Toxizität und zur klinischen Wirksamkeit der Medikamente führen. Mit einem Überblick über die derzeitigen Kenntnisse in der klinischen Pharmakologie dieser wichtigen Stoffgruppe hoffen wir, dem praktischen Tierarzt eine wirklichkeitsgetreue wissenschaftliche Grundlage für deren sichere und effektive Anwendung in der Pferdeheilkunde geben zu können.
\end{abstract}

beträchtliche Bedeutung für den praktischen Tierarzt. Entsprechend sollte sich der Kliniker im klaren darüber sein, daß pharmakologische Werte, die aus Messungen der Wirkungen eines speziellen Arzneimittels bei einer bestimmten Tierart stammen, nicht in jedem Fall auf andere übertragbar sind. Die Eliminations-Halbwertszeit von Phenylbutazon beträgt $\mathrm{z}$. B. beim Pferd 3,5 bis $8 \mathrm{~h}$ je nach verabreichter Dosis (Piperno, Ellis, Getty und Brody, 1968). Die Halbwertszeit wird desto länger, je größer die applizierte Menge ist. Ähnliche Halbwertszeitangaben liegen vor für das Kaninchen ( $3 \mathrm{~h}$ ), den Hund ( $6 \mathrm{~h}$ ) und das Meerschweinchen (5 h) (Ruckebusch und Toutain, 1983). Beim Menschen und beim Rind ist sie dagegen wesentlich länger (72 bzw. $40 \mathrm{~h}$ ) (Snow, 1981). Daher müssen sich auch Arbeiten in der Grundlagenforschung, bei denen die Wirkungsdauer der NSAIDs zu bestimmen ist, auf die jeweilige Zielspezies beziehen.

\section{Historischer Hintergrund}

In den vergangenen sieben Jahren sind mehrere Übersichtsdarstellungen und Artikel über NSAIDs beim Pferd erschienen (Jeffcott und Colles, 1977; Jones und Hamm, 1977; Tobin, 1979 a, b; Snowe, 1981). In der Zwischenzeit ist eine ganze Menge neuer Information hinzugekommen. Manches davon widerspricht sich gegenseitig, größtenteils hat sie jedoch entscheidende Bedeutung für die sichere und ef- 


\section{CARBOXYSÄUREN \\ $\mathrm{R}-\mathrm{COOH} \rightleftharpoons \mathrm{R}-\mathrm{COO}^{-}+\mathrm{H}^{+}\left(\mathrm{Na}^{+}\right)$}
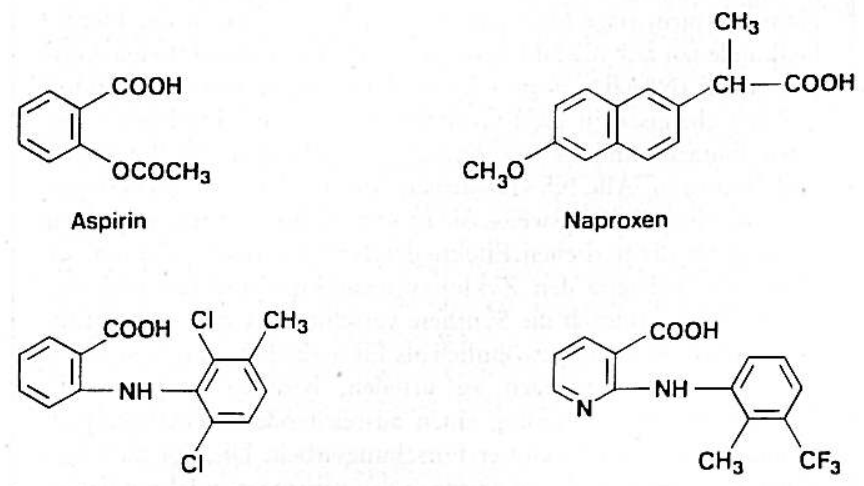

Meclofensäure

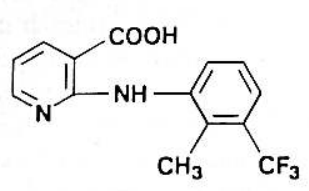

Flunixin

Abb. 1a: Strukturformeln der carbonsauren steroidfreien Entzündungshemmer, die in der Pferdeheilkunde in Gebrauch sind.

fektive Anwendung von NSAIDs bei der Tierart Pferd. Ein Hauptmotivationspunkt war der Bericht von Snow, Bogan, Douglas und Thompson (1979), die herausfanden, daß Phenylbutazon bei Ponies in Dosierungen hoch toxisch wirken kann, die nur etwas über den vom Hersteller empfohlenen liegen.

Sowohl in der Humanmedizin als auch in der Veterinärmedizin sind NSAIDs aber seit langem in Gebrauch. Seit Jahrhunderten kennt man die medikamentösen Eigenschaften von Weidenrinde (Salix). Ihre erfolgreiche Anwendung bei der Heilung der Malaria (Fieber) beschrieb Pfarrer Stone aus Chipping Norton der Royal Society of London im Jahre 1763. Einige Jahre später wies Leroux als wirksamen Inhaltsstoff von Salix Salicin, ein bitteres Glycosid, nach. Bei der Hydrolyse setzt Salicin in Anwesenheit von Ätzkali Glukose und Salicylalkohol (Saligenin) frei. Die ihm verwandte Säure, Salicylsäure, wurde erstmals 1838 aus Saligenin gewonnen, und die erste Behandlung von rheumatischem Fieber mit Hilfe von Natriumsalicylat beim Menschen führte man 1875 durch.

Die Anwendung in der Veterinärmedizin folgte bald darauf. Dun (1895) bekundete die Ansicht, die Salicylate wirkten „direkt auf die trophischen Nerven und die Nervenzentren" und "verzögerten die Oxidation von Protoplasmen". Salicylate erwiesen sich angeblich als „besonders gute Dienste leistend bei der Bekämpfung von Fieber und Schmerzzuständen des akuten Rheumatismus“. Natriumsalicylat kam auf Grund der fehlenden Reizwirkungen der Salicylsäure bevorzugt zur klinischen Anwendung. Dun (1895) berichtete, daß „Mr. E. Price, Birmingham, zufriedenstellende Effekte bei Pferden erzielte; er verordnete 10 Körner ( 1 Korn $\triangleq 64,8 \mathrm{mg}$ ) alle zwei Stunden, nach und nach ansteigend bis zu einer Drachme $(\hat{=} 3,88 \mathrm{~g})$; seiner Aussage gemäß waren die rheumatischen Schmerzen nach 48 Stunden verschwunden“. Die Einführung des Essigsäureesters der Salicylsäure, Acetylsalicylsäure (Aspirin), kam 1899. In unserem Jahrhundert wurden immer wieder synthetische Verbindungen mit notwendigerweise ähnlichen Wirkun-

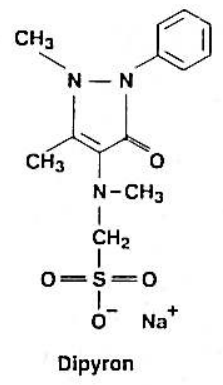

ENOLSÄUREN

$$
\mathrm{R}-\mathrm{C}=\mathrm{O} \rightleftharpoons \mathrm{R}=\mathrm{C}-\mathrm{OH} \rightleftharpoons \mathrm{R}=\mathrm{C}-\mathrm{O}^{-}+\mathrm{H}^{+}\left(\mathrm{Na}^{+}\right)
$$
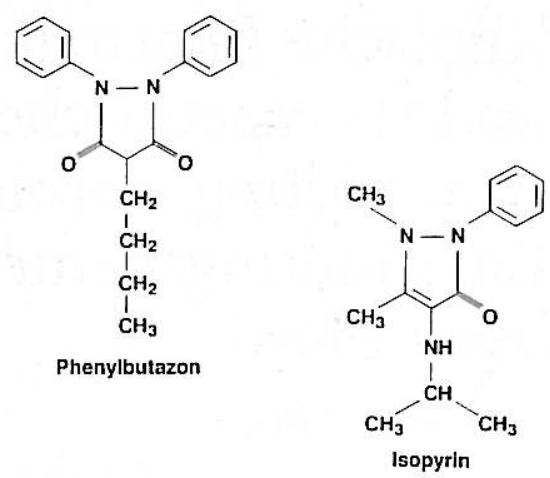

Abb. 1b: Strukturformeln der enolsauren steroidfreien Entzündungshemmer, die in der Pferdeheilkunde in Gebrauch sind.

gen und Nebenwirkungen neu zur Therapie herangezogen. Heute steht eine ganze Reihe von Stoffen für die klinische Anwendung zur Verfügung. Die Suche nach dem idealen Entzündungshemmer dauert an, aber einige Fortschritte wurden in den letzten Jahren erzielt.

\section{Steroidfreie Entzündungshemmer}

\section{Chemische Einteilung}

Die NSAIDs lassen sich chemisch in zwei Gruppen einteilen, die Carbonsäuren und die Enolsäuren. Letztere kommen in zwei isomeren Formen vor (Keto- und Enolform). Das Wasserstoffatom der Carboxylgruppe oder des EnolIsomers reagiert mit Basen und bildet so beispielsweise $\mathrm{Na}$ triumsalze (Abb. 1 a, b).

Unterschiede in der chemischen Struktur erlauben eine weitere Einordnung in mehrere Untergruppen, wie Abb. 2 zeigt. Die Abbildungen $1 \mathrm{a}$ und $\mathrm{b}$ geben eine Darstellung der Strukturformeln jener Verbindungen, die gegenwärtig im United Kingdom als NSAIDs zum Gebrauch in der Pferdeheilkunde zugelassen sind. Die von den Herstellern empfohlenen Dosierungen sind in Tabelle 1 und 2 aufgeführt. Derzeit sind verschiedene Medikamente für die Therapie in der Veterinärmedizin erhältlich. Die mitgelieferten Angaben sind aber oft spärlich, wenn es sie überhaupt gibt. Aus diesem Grund befaßt sich die vorliegende Übersicht nur mit solchen Arzneimitteln, für die zuverlässige Information zur Verfügung steht.

\section{Wirkungsweise}

Der Wirkungsmechanismus der NSAIDs besteht in der Hemmung des Zyklooxygenase-(Prostaglandin-Synthetase-)

Enzymstoffwechsels (Higgs, Palmer, Eakins und Moncada, 1981), wie Higgins und Lees (1984) es in Einzelheiten in einer früheren Übersicht besprochen haben. Über den exakten Angriffspunkt der NSAIDs und die Art, in der sie es tun, ist noch nichts bekannt. Dagegen ist den Autoren der 
Tab. 1: Rezepturformeln und von den Herstellern empfohlene Dosierungen der zur oralen Anwendung im United Kingdom zugelassenen NSAIDs

\begin{tabular}{|c|c|c|c|c|}
\hline Inhaltsstoffe & $\begin{array}{l}\text { Handelsname } \\
\text { (Hersteller) }\end{array}$ & $\begin{array}{l}\text { Rezeptur und } \\
\text { Darreichungsform }\end{array}$ & $\begin{array}{l}\text { Dosierung pro } \\
\text { Verpackungseinheit }\end{array}$ & $\begin{array}{l}\text { Empfohlene Tagesdosis } \\
\text { (mg/kg KG, falls nicht } \\
\text { anders angegeben) }\end{array}$ \\
\hline Phenylbutazon & $\begin{array}{l}\text { Equipalazone } \\
\text { (Arnolds) }\end{array}$ & Pulver/Päckchen & 1,0 g/Päckchen & $\begin{array}{l}\text { Pferd: } 4,4 \text { zweimal am 1. Behand- } \\
\text { lungstag, dann } 4 \text { Tage lang } 2,2 \\
\text { zweimal täglich, danach } 2,2 \text { einmal } \\
\text { täglich } \\
\text { Pony: } 4,4 \text { einmal täglich } 4 \text { Tage } \\
\text { lang, dann } 4,4 \text { jeden } 2 \text {. Tag }\end{array}$ \\
\hline Phenylbutazon & $\begin{array}{l}\text { Prodynam Powder } \\
\text { (Leo) }\end{array}$ & Pulver/Päckchen & 1,0 g/Päckchen & $\begin{array}{l}4 \text { Tage lang } 4,2 \text { zweimal täglich, } \\
\text { danach halbe Initialdosis und wei- } \\
\text { ter kleiner werdende Dosen, bis die } \\
\text { niedrigst mögliche noch wirksame } \\
\text { Dosierung erreicht ist }\end{array}$ \\
\hline Phenylbutazon & $\begin{array}{l}\text { Buvetzone Bolus } \\
\text { (Willows Francis) }\end{array}$ & $\begin{array}{l}\text { Bolus/zur Dosie- } \\
\text { rung markiert }\end{array}$ & $2,0 \mathrm{~g} /$ Bolus & $\begin{array}{l}3 \text { bis } 4 \text { Tage lang } 4,0 \mathrm{~g} \text { pro Pferd } \\
\text { täglich, danach } 4 \text { Tage lang } 2,0 \\
\text { täglich und dann } 1,0 \text { täglich }\end{array}$ \\
\hline Phenylbutazon & $\begin{array}{l}\text { Equipalazone } \\
\text { Paste E-PP } \\
\text { (Arnolds) }\end{array}$ & Paste & 1,0 g/Dosierungseinheit & wie bei Equipalazone Pulver \\
\hline Phenylbutazon & $\begin{array}{l}\text { Phenyzone Oral } \\
\text { Paste } \\
\text { (C-Vet) }\end{array}$ & Paste & 1,0 g/Dosierungseinheit & $\begin{array}{l}4 \text { Tage lang } 6,6 \text { zweimal täglich, } \\
\text { danach } 4 \text { Tage lang } 4,6 \text { täglich, da- } \\
\text { nach } 4 \text { Tage lang } 2,3 \text { jeden } 2 \text {. Tag }\end{array}$ \\
\hline Phenylbutazon & $\begin{array}{l}\text { Phenylbutazone } \\
\text { Tablets BP } \\
\text { (Veterinary Drug Co) }\end{array}$ & Tablette & 200 mg/Tablette & 4 bis 8 \\
\hline $\begin{array}{l}\text { Phenylbutazon plus } \\
\text { Chinchopen }\end{array}$ & $\begin{array}{l}\text { Buta-Leukotropin } \\
\text { (Berk) }\end{array}$ & Pulver/Päckchen & $\begin{array}{l}500 \text { mg Phenylbutazon/ } \\
\text { Päckchen } 1,0 \mathrm{~g} \text { Chin- } \\
\text { chopen/Päckchen }\end{array}$ & $\begin{array}{l}4 \text { Tage lang } 4,4 \text { (Phenylbutazon) } \\
\text { auf mehrere Gaben verteilt, dann } \\
4 \text { Tage lang } 2,2 \text { Einzeldosen und } \\
\text { danach } 12 \text { Tage lang } 1,1 \text { jeden } 2 . \\
\text { Tag }\end{array}$ \\
\hline Flunixin meglumin & $\begin{array}{l}\text { Finadyne granules } \\
\text { (Fisons) }\end{array}$ & Granulat/Päckchen & 250 mg/Päckchen & $\begin{array}{l}\text { an bis zu } 5 \text { aufeinanderfolgenden } \\
\text { Tagen } 1,1 \text { täglich }\end{array}$ \\
\hline Meclofensäure & $\begin{array}{l}\text { Arquel granules } \\
\text { (Parke Davis) }\end{array}$ & Granulat/Päckchen & 500 mg/Päckchen & $\begin{array}{l}5 \text { bis } 7 \text { Tage lang } 2,2 \text { täglich, nach } \\
7 \text { Tagen } 2,2 \text { täglich oder in größe- } \\
\text { ren Abständen }\end{array}$ \\
\hline Naproxen & $\begin{array}{l}\text { Equiproxen } \\
\text { Granules } \\
\text { (Syntex) }\end{array}$ & Granulat/Päckchen & 4,0 g/Päckchen & $\begin{array}{l}\text { an bis zu } 14 \text { aufeinanderfolgenden } \\
\text { Tagen } 10 \text { täglich }\end{array}$ \\
\hline
\end{tabular}

Nachweis gelungen, daß sowohl Phenylbutazon als auch Flunixin, als intravenöse Einzelgabe in therapeutischer Dosierung an Ponies verabreicht, 12 bis 24 h lang die Bildung von Prostaglandin $\mathrm{E}_{2}$ in entzündlichen Exsudaten vermindert (Higgins und Lees, 1983; Higgins, Lees und Taylor, 1984; Lees und Higgins, 1984). Mit Hilfe dieser Befunde läßt sich wohl die Beobachtung erklären, daß die klinische Reaktion auf diese Wirkstoffe nach einer Einzelgabe länger als $24 \mathrm{~h}$ oder nach der letzten Applikation in einer Reihe von Behandlungen sogar 3 Tage lang anhält (Scott Dunn, 1971). Die Ergebnisse der klinischen und auch der Laboruntersuchung mögen überraschen, wenn man die kurze Halbwertszeit dieser Medikamente bei Pferden, 4,5 h bei Phenylbutazon und 1,6 h bei Flunixin, betrachtet. Die Diskrepanz zwischen kurzer Halbwertzeit und langer Wirkungsdauer läßt sich mit der Feststellung erklären, daß viele NSAIDs irreversible Bindungen mit der Zyklooxygenase eingehen. Dies führt dazu, daß wohl am Entzündungsort erst neue Enzyme synthetisiert werden müssen, bevor weitere Eikosane entstehen können. Meclofensäure, Aspirin und Phenylbutazon sollen angeblich einen irreversiblen Antagonismus des Zyklooxygenase-Stoffwechsels bewirken, während der Reaktionsmechanismus von Oxyphenbutazon offenbar reversibel ist.

Diese pharmakodynamische Hypothese erklärt vielleicht die relativ lange Wirkdauer der NSAIDs nicht ausreichend. Man hat nun aber auch beobachtet, daß nach $12 \mathrm{~h}$ die Flunixin- und Phenylbutazonkonzentrationen im entzündlichen Exsudat höher waren als im Plasma, so daß die Spiegel in entzündeten Geweben, auf welche die Plasmakonzentrationen schließen lassen, zu niedrig sind. Dies ist als pharmakokinetische Erklärung des Phänomens denkbar (Higgins et al., 1984; Lees und Higgins, 1984). Gleich ob Verhältnisse entsprechend der dynamischen oder der kinetischen Hypothese oder beidem für die lange Dauer des Effekts der NSAIDs verantwortlich sind, in jedem Fall ist die 
Tab. 2: Von den Herstellern empfohlene Dosierungen und Applikationsarten der zur parenteralen Verabreichung im United Kingdom zugelassenen NSAIDs

\begin{tabular}{|c|c|c|c|c|}
\hline Inhaltsstoffe & $\begin{array}{l}\text { Handelsname } \\
\text { (Hersteller) }\end{array}$ & $\begin{array}{l}\text { Konzentration } \\
(\mathrm{mg} / \mathrm{ml})\end{array}$ & $\begin{array}{l}\text { Empfohlene Tagesdosis } \\
\text { (mg/kg falls nicht anders angegeben) }\end{array}$ & Applikationsart \\
\hline Phenylbutazon & $\begin{array}{l}\text { Intrazone } \\
\text { (Arnolds) }\end{array}$ & 200 & $\begin{array}{l}4,4 \text { als einmalige Gabe } \\
\text { (nachfolgend orale Therapie) }\end{array}$ & iv Injektion \\
\hline Phenylbutazon & $\begin{array}{l}\text { Phenyzone } \\
\text { Injection } \\
\text { (C-Vet) }\end{array}$ & 200 & $\begin{array}{l}2,2 \text { bis } 4,4 \text { an höchstens } \\
5 \text { aufeinanderfolgenden Tagen }\end{array}$ & iv Injektion \\
\hline $\begin{array}{l}\text { Phenylbutazon plus } \\
\text { Prednisolon }\end{array}$ & $\begin{array}{l}\text { Azoid } \\
\text { (Arnolds) }\end{array}$ & $\begin{array}{l}200 \text { Phenylbutazon } \\
2 \text { (Prednisolon) }\end{array}$ & $\begin{array}{l}4,4 \text { (Phenylbutazon) als einmalige Gabe } \\
\text { (nachfolgend orale Therapie) }\end{array}$ & iv Injektion \\
\hline $\begin{array}{l}\text { Phenylbutazon plus } \\
\text { Isopyrin }\end{array}$ & $\begin{array}{l}\text { Tomanol } \\
\text { (Intervet) }\end{array}$ & $\begin{array}{l}120 \text { (Phenylbutazon) } \\
240 \text { (Isopyrin) }\end{array}$ & 4,2 (Phenylbutazon) 1 bis 4 Tage lang & iv Injektion \\
\hline Flunixin meglumin & $\begin{array}{l}\text { Finadyne } \\
\text { Solution } \\
\text { (Fisons) }\end{array}$ & 50 & 1,1 täglich bis zu 5 Tage lang & iv oder im Injektion \\
\hline $\begin{array}{l}\text { Dipyron plus } \\
\text { Hyoscin N-butylbromid }\end{array}$ & $\begin{array}{l}\text { Buscopan } \\
\text { Compositum } \\
\text { (Boehringer Ingel- } \\
\text { heim) }\end{array}$ & $\begin{array}{l}500 \text { (Dipyron) } \\
4 \text { (Hyoscin) }\end{array}$ & $15 \mathrm{~g}$ Dipyron pro Pferd & iv oder im Injektion \\
\hline $\begin{array}{l}\text { Dipyron plus } \\
\text { Methindizathydrochlorid }\end{array}$ & $\begin{array}{l}\text { Isaverin } \\
\text { (Bayer) }\end{array}$ & $\begin{array}{l}250 \text { (Dipyron) } \\
2,5 \text { (Methindizat) }\end{array}$ & $\begin{array}{l}11,1 \text { (Dipyron) bei Bedarf } \\
\text { Wiederholung nach } 4 \text { bis } 6 \text { Stunden }\end{array}$ & iv oder im Injektion \\
\hline $\begin{array}{l}\text { Chinchopen plus } \\
\text { Natriumsalicylat } \\
\text { plus Hexamin }\end{array}$ & $\begin{array}{l}\text { Leucotropin } \\
\text { Injection } \\
\text { (Berk) }\end{array}$ & $\begin{array}{l}80 \text { (Chinchopen) } \\
30 \text { (Salicylat) } \\
240 \text { (Hexamin) }\end{array}$ & $\begin{array}{l}17,8 \text { (Chinchopen) und } \\
6,7 \text { (Salicylat) täglich }\end{array}$ & $\begin{array}{l}\text { iv oder subkutane } \\
\text { Injektion }\end{array}$ \\
\hline $\begin{array}{l}\text { Chinchopen plus } \\
\text { Natriumsalicylat plus } \\
\text { Hexamin plus Predni- } \\
\text { solon-m-sulphobenzoat }\end{array}$ & $\begin{array}{l}\text { Predno- } \\
\text { Leucotropin } \\
\text { Injection } \\
\text { (Berk) }\end{array}$ & $\begin{array}{l}80 \text { (Chinchopen) } \\
30 \text { (Salicylat) } \\
240 \text { (Hexamin) } \\
1 \text { (Prednisolon) }\end{array}$ & $\begin{array}{l}17,8 \text { (Chinchopen) und } \\
6,7 \text { (Salicylat) täglich }\end{array}$ & $\begin{array}{l}\text { iv oder subkutane } \\
\text { Injektion }\end{array}$ \\
\hline
\end{tabular}

iv intravenös

im intramuskulär

Plasma-Wirkstoffkonzentration wahrscheinlich nicht zu jedem vorgegebenen Zeitpunkt ein zweckmäßiger Leitfaden für die therapeutische Aktivität.

\section{Wirksamkeit}

An Labortieren hat man untersucht, wie hoch die für eine 50prozentige Hemmung der Zyklooxygenase-Aktivität erforderlichen Dosen sind. Nach Stärke der Wirksamkeit geordnet, ergab sich die Reihenfolge: Meclofensäure > Indomethacin $>$ Naproxen $>$ Phenylbutazon $>$ Aspirin (Vane, 1973; Higgs et al., 1981). Eine ähnliche stärkemäßige Anordnung stellte sich bei der Messung der entzündungshemmenden Wirkung im carrageenininduzierten Pfotenödem von Ratten heraus (Vane, 1973; Flower, 1974). Dieselbe Reihenfolge trifft auch für die Dosierungen zu, derer es beim Menschen bedarf, um einen klinischen Effekt zu erreichen. Den für die Anwendung beim Pferd empfohlenen Gaben nach zu urteilen ist die Rangordnung hinsichtlich Wirksamkeit vermutlich im wesentlichen dieselbe (Meclofensäure $>$ Phenylbutazon $>$ Naproxen $>$ Salicylat). Flunixin steht in der Stärkeskala des Pferds an erster Stelle, noch vor Meclofensäure, ist aber für praktische Ärzte nicht erhältlich. Es zeigt sich, daß Phenylbutazon seinen Platz in der Wirksamkeitsliste des Pferds verbessert hat. Der Grund hierfür liegt wahrscheinlich darin, daß beim Pferd mit einem Plasmaspiegel von 5 bis $15 \mu / \mathrm{ml}$ ein therapeutischer
Effekt zu erzielen ist (Jenny, Steinijans und Seifert, 1979; Gerring, Lees und Taylor, 1981). Beim Menschen sind hingegen offenbar Plasmakonzentrationen von 50 bis $150 \mu / \mathrm{ml}$ erforderlich (Kampmann und Frey, 1966).

Diese Unterschiede lassen sich wohl einem laut Bericht größeren proteingebundenen Anteil beim Menschen (98,8 Prozent) als beim Pferd (96 Prozent) zuschreiben (Snow, 1981). Dieser kleinen Differenz in der prozentualen Bindung hat man ursprünglich Bedeutung zugemessen, denn die "freie" Plasmakonzentration von Phenylbutazon, 4 Prozent der Gesamtmenge beim Pferd und 1,2 Prozent beim Menschen, mag in $\mu / \mathrm{ml}$ ausgedrückt etwa gleich sein. Dann fand sich aber eine über 99prozentige Bindung von Phenylbutazon an das Plasmaprotein beim Pferd (Gerring et al., 1981). Im Lichte dieser Erkenntnis lassen sich die relativ niedrigen erforderlichen Dosen beim Pferd eher mit bestehenden speziesspezifischen Abweichungen in der Struktur des Zyklooxygenase-Enzyms erklären. Unter Umständen besitzt das Enzym in Geweben vom Pferd eine niedrigere Konstante hinsichtlich Hemmung durch Phenylbutazon.

Unglücklicherweise findet häufig die Tatsache nicht genug Beachtung, daß die Wirksamkeit als solche überhaupt keinen Vorteil eines NSAIDs gegenüber einem anderen bedeutet. Praktische Tierärzte sollten jede anderslautende Angabe zu einem Präparat mit Vorsicht genießen. Erhöhte 


\section{STEROIDFREIE ENTZÜNDUNGSHEMMER}

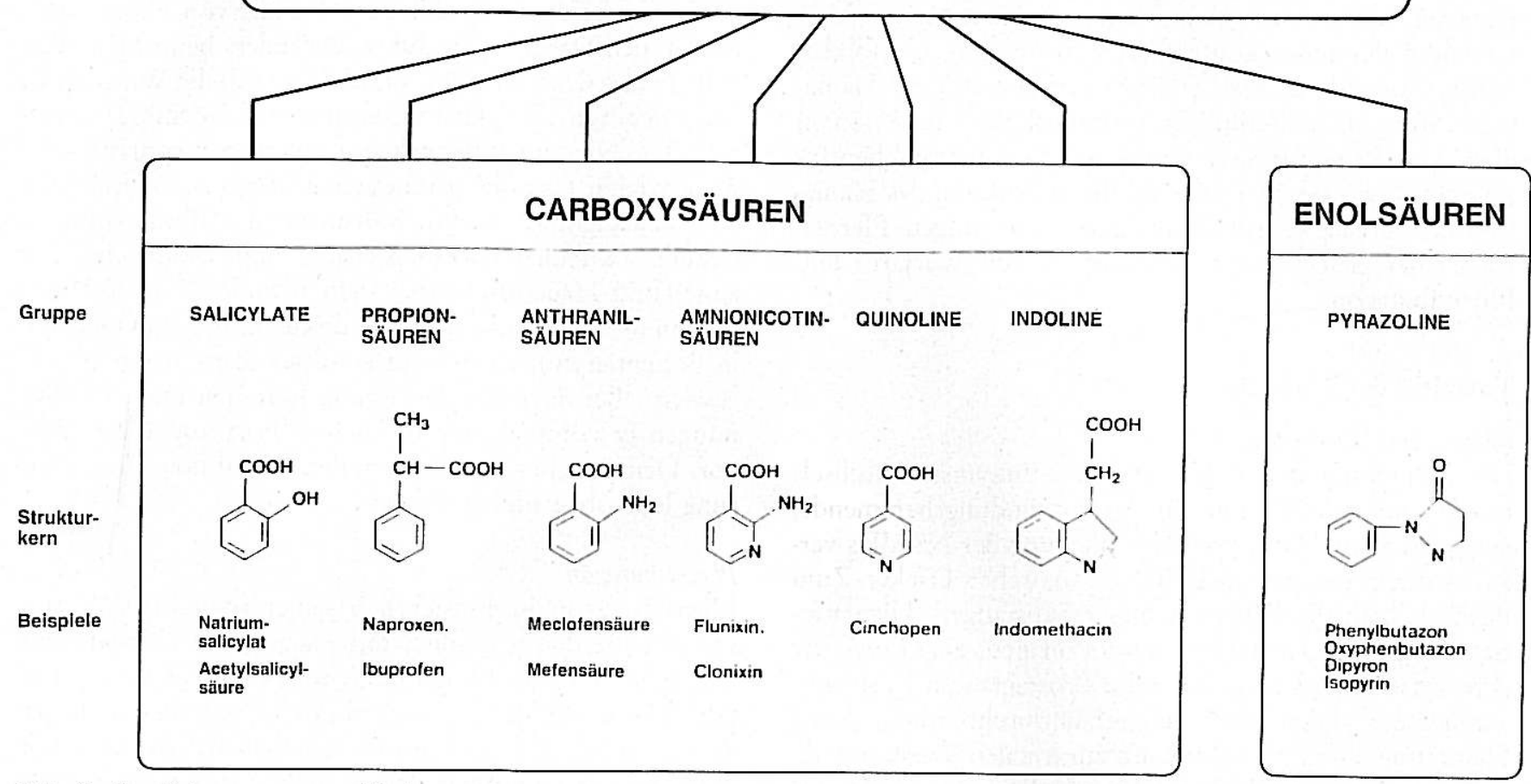

Abb. 2: Klassifizierung von steroidfreien entzündungshemmenden Medikamenten.

Wirkungsstärke ist nicht gleichzusetzen mit erhöhter Effektivität; letztere bestimmt das Stärke-Toxizität-Verhältnis (siehe unten).

\section{Wecbselwirkungen}

Einige Pferdepraktiker haben anscheinend die recht stark in Mode gekommene Vorgehensweise übernommen, bei einer Reihe klinischer Zustandsbilder zwei oder mehr NSAIDs kombiniert zu verabreichen. Außerdem werden NSAIDs häufig zusammen mit Stoffen aus anderen Arzneimittelklassen wie den Antibiotika verschrieben. Zur Überlegung, NSAIDs therapeutisch bei Pferden anzuwenden, gehört immer, das Auftreten und die möglichen Gefahren von Wechselwirkungen zwischen Arzneimitteln zu berücksichtigen. Generell addiert sich die Wirkung zweier gemeinsam applizierter NSAIDs. Da ihr Wirkungsmechanismus derselbe ist, nämlich die Hemmung des Zyklooxygenase-Enzyms, findet sich schwerlich ein zugkräftiges Argument zugunsten einer kombinierten Anwendung. Eine höhere Dosis eines einzelnen Medikaments führt vermutlich zum selben Effekt.

In einer handelsmäßig angebotenen Wirkstoff-Kombination, die Phenylbutazon und Isopyrin enthält (Tomanol; Intervet), haben die NSAIDs, die beide zum Enol-Typ gehören, eine synergistische Wechselwirkung. Diese hat ihren Grund nicht in einer erhöhten Empfindlichkeit der Rezeptoren, sondern in der verlängerten Halbwertszeit der Wirkstoffe. Bei getrennter Applikation bei Pferden beträgt, so zeigte sich, die Halbwertszeit 6,2 bzw. 3,8 h für Phenylbutazon bzw. Isopyrin. In dem Kombinationspräparat lag sie bei 10,2 h für Phenylbutazon und 5,0 h für Isopyrin
(Jenny et al., 1979). Die Halbwertszeit von Phenylbutazon war bei dieser Untersuchung um 65 Prozent erhöht. Die Ursache dieser Steigerung ist vermutlich in einer gegenseitigen Herabsetzung der Geschwindigkeit, mit der die Leber des Pferds beide Substanzen verstoffwechselt, zu sehen. Hinzu kommt, daß bisher die Dosierung von Phenylbutazon in Tomanol doppelt so hoch war wie die empfohlene Dosis für allein verabreichtes Phenylbutazon. Daher überrascht es wohl nicht, daß das Präparat im Ruf stand, klinisch eine starke und langanhaltende Wirkung zu besitzen. Der Synergismus läßt diese Wirkstoffkombination eigentlich in keiner Weise vorteilhaft gegenüber anderen erscheinen, und es wäre denkbar, daß Phenylbutazon allein in hohen Dosen gleich intensive Effekte hat. Nichtsdestoweniger führen Unterschiede in der Wirkung der beiden Arzneimittel möglicherweise zur gesteigerten Effizienz der Kombinationspräparate; Phenylbutazon ist hauptsächlich ein Entzündungshemmer, wogegen Isopyrin mehr analgetisch wirkt.

Eine weitere mögliche Grundlage für Wechselwirkungen zwischen NSAIDs und anderen pharmakologisch wirksamen Substanzen hat als Kernpunkt die Verdrängung eines Wirkstoffs aus seinen Bindungsstellen an Plasmaproteinen durch einen anderen. Verabreicht man einem Pferd Phenylbutazon, das bereits mit einem anderen Arzneimittel mit hohem Proteinbindungsvermögen vorbehandelt ist, so steigt die freie Konzentration der ersten Substanz. Eine größere Menge von ihr kann dann pharmakologische und eventuell auch toxische Effekte ausüben. Warfarin bindet sich beispielsweise in hohem $\mathrm{Maß}$ an Plasmaprotein, und die Verdrängung von seinen Bindungsstellen durch Phenyl- 
butazon odere andere NSAIDs könnte theoretisch zur Warfarin-Toxizität führen. Eine solche kombinierte Anwendung sollte daher, wenn überhaupt, mit großer Vorsicht und nur unter kontrollierten Bedingungen erfolgen. Traub, Paulsen und Reed (1983 b) berichteten über Hämatombildung im Anschluß an intramuskuläre Injektionen, über Austritt von seröser Flüssigkeit aus chirurgischen Inzisionsstellen und über eine deutliche Senkung des Hämatokrits und der Thrombozytenzahl bei einigen Pferden nach einer gleichzeitigen Applikation von Warfarin und Phenylbutazon.

\section{Toxizität der NSAIDs}

\section{Allgemeine Überlegungen}

Die Hemmung des Zyklooxygenase-Enzymstoffwechsels ist vermutlich nicht nur für die entzündungshemmende, analgetische und antipyretische Wirkung der NSAIDs verantwortlich, sondern auch für ihre toxischen Effekte. Zum Beispiel kann die Fähigkeit dieser Substanzen, Ulzerationen im Magen-Darm-Trakt hervorzurufen, eine Folge der Prostaglandin- $\mathrm{E}_{2}-$ Hemmung sein; Prostaglandin $\mathrm{E}_{2}$ steigert anscheinend lokal die Schleimhautdurchblutung. Seine Hemmung durch NSAIDs kann zur lokalen Vasokonstriktion, Hypoxie und Nekrose führen. Wenn nun aber die Unterbrechung gleicher biochemischer Stoffwechselwege der Grund sowohl für die therapeutischen als auch für die toxischen Wirkungen der NSAIDs ist, dann wäre die Erwartung berechtigt, daß bei gleich wirksamer Dosierung alle Medikamente mit derselben Wahrscheinlichkeit auch die toxischen Effekte hervorrufen. Wenn ein Wirkstoff $10 \mathrm{mal}$ so stark wie ein anderer ist, sollte er auch $10 \mathrm{mal}$ so toxisch wirken. Tatsächlich bestätigte sich diese Hypothese weder bei experimentellen Untersuchungen noch stimmt sie mit klinischen Beobachtungen überein. Bei Ratten hat zum Beispiel Aspirin in entzündungshemmenden Dosen eine hohe Ulzerogenität, Salicylat dagegen eine niedrige und Naproxen eine mittlere (Whittle et al., 1980). Möglicherweise sind die Erklärung hierfür geringe Strukturunterschiede bei den Zyklooxygenase-Enzymen an verschiedenen Körperstellen und in unterschiedlichen Geweben.

Besonders interessiert an einer selektiven ZyklooxygenaseHemmung sind Forscher, die versuchen, ein nichtulzerogenes NSAID zu entwickeln. Dieses NSAID soll gegenüber der Zyklooxygenase im Magen-Darm-Trakt nur schwach, aber hoch wirksam gegenüber dem Enzym an akut und chronisch entzündeten Stellen sein. Das AminopyrazolinDerivat BW755C entspricht diesem Wirkungs-VerteilungsMuster (Higgs, Flower und Vane, 1979).

Es ist nicht verwunderlich, daß aus Berichten Nebenwirkungen bei Patienten (besonders beim Menschen) nach einer NSAID-Therapie in einem weiten Bereich bekannt sind. Denn die zur Eikosan-Bildung befähigten Enzyme sind sehr verbreitet (alle untersuchten Körpergewebe mit Ausnahme der Erythrozyten), und ihre physiologischen Funktionen sind wahrscheinlich vielfältiger Natur. Andererseits überrascht vielleicht, daß einige dieser Nebeneffekte sehr selten auftreten. Die am häufigsten beobachtete $\mathrm{Ne}$ benwirkung besteht in einer Reizung des Magen-Darm-
Trakts sowohl bei Patienten in der Human- als auch in der Veterinärmedizin und in einer Schädigung, die zu Magenoder Darmgeschwüren und zum Verlust von Plasma oder Blut in den Darm hinein führt. Besonders beim Menschen trifft man jedoch auch oft auf andere toxische Wirkungen. Dazu gehören Flüssigkeitsretention und Ödeme, Nephrotoxizität (Nierenpapillennekrose, tubuläre Nephritis), Hepatotoxizität (sowohl parenchymatöse als auch cholestatische Schädigungsformen), Störungen der Blutzusammensetzung (Knochenmarksdepression und aplastische Anämie) und Hautausschläge. Auch pathologische Veränderungen der Kreislauf-, Atem- und Nierenfunktion tauchten in Berichten auf, ebenso ein gestörtes Säure-Basen-Gleichgewicht. Bei normaler Dosierung kommen diese Erscheinungen gewöhnlich nur in leichter Form oder gar nicht vor. Dennoch können sie in Fällen von akuter Überdosierung lebensbedrohlich werden.

\section{Phenylbutazon}

Phenylbutazon ist hinsichtlich seiner toxischen Wirkungen bei Pferden gründlich untersucht. Die Aufmerksamkeit richtete sich stark auf das Medikament, als Snow et al. (1979) vom Tod dreier Shetland-Ponies berichteten, die das Arzneimittel 14 Tage lang oral erhalten hatten. Bis dahin hatte man angenommen, daß Phenylbutazon beim Pferd in bezug auf die Dosierung einen großen Sicherheitsspielraum hat. Es war nicht nur über 30 Jahre lang verbreitet in Gebrauch, sondern oft hatten einzelne Pferde niedrige Dosen (2,2 bis $5 \mathrm{mg} / \mathrm{kg}$ Körpergewicht [KG]/Tag) als Dauermedikation über einen Zeitraum von mehreren Monaten hinweg verordnet bekommen (Scott Dunn, 1971; Gabel, Tobin, Ray und Maylin, 1977). Zwar lagen auch ein paar Berichte in der Literatur vor über eine vermutete Toxizität. Darin waren niedriger Hämatokrit (Roberts, 1982), hypoplastische Anämie (Dunavant und Murray, 1975), Kolik, Nasenbluten und Ulcusbildung im Darm (Gabriel und Martin, 1962; Hopes, 1972) und nekrotisierende Phlebitis der kleinen Portalvenen (Gabriel und Martin, 1962) erwähnt. Es war aber keineswegs erwiesen, daß Phenylbutazon in $\mathrm{Zu}$ sammenhang mit all diesen Reaktionen stand. Die relativ kurze Halbwertszeit von Phenylbutazon beim Pferd verglichen mit der beim Menschen gab Anlaß zur Vermutung, $\mathrm{da} ß$ eine Therapie mit ein- bis zweimaliger Verabreichung pro Tag wahrscheinlich nicht zu einer Kumulation des Wirkstoffs im Körper und zum Erreichen toxischer Spiegel führen würde.

Snow et al. (1979) zeigten, daß Phenylbutazondosen, die etwas über den zu dieser Zeit empfohlenen lagen ( 8,1 bis 14,6 $\mathrm{mg} / \mathrm{kg} \mathrm{KG}$ täglich gegenüber $8,8 \mathrm{mg} / \mathrm{kg} \mathrm{KG}$ täglich) und länger ( 8 bis 14 Tage gegenüber 4 Tagen plus einer Entwöhnungsdosis) appliziert wurden, akut toxisch für Ponies waren. Als Symptome traten Anorexie, Mattigkeit, Ascites, orale Ulzerationen und Ödeme in der Dickdarmwand auf. Mit großer Wahrscheinlichkeit trugen die hohen Dosierungen zu der toxischen Wirkung bei, und die Gabe des Arzneimittels jeden Tag als Einzeldosis verstärkte noch die Erscheinungen. In einer Folgestudie bestätigten sich die Befunde (Snow et al., 1981 b). Dabei erhielten 5 Ponies einer Shetland-Kreuzungsrasse Phenylbutazon in relativ hoher 
Dosierung (12 mg/kg KG täglich), aber diesmal auf mehrere Tagesgaben verteilt, 8 bis 10 Tage lang.

Wieder waren klinisch und pathophysiologisch Anzeichen von Toxizität festzustellen. Es trat eine Senkung der Plasmaprotein- und Albuminkonzentration auf, und zwei Ponies starben. Bei der Sektion waren massive Ulzerationen im Darm, hauptsächlich im Colon ascendens und Caecum, sichtbar. Einige Geschwüre fanden sich auch in der Maulhöhle, jedoch nur bei einem einzigen Pony ein paar im Duodenum und im Magen. Als Todesursache betrachtete man eine Darmerkrankung mit Proteinverlust, die bei entsprechend schwerem Verlauf zu einer Abnahme des Blutvolumens, Hämokonzentration, zum hypovolämischen Schock und zum Kreislaufversagen führen kann. Wenn das ulzerative Geschehen ernste Formen annimmt, wird vermutlich unter Umständen das Schocksyndrom noch durch eine Sepsis verstärkt.

Auch Roberts (1981) beschrieb einen Fall von Hypoproteinämie, Facialödem und Ruptur des Colon ascendens bei einem Tier, das unterschiedlich hohe Dosen Phenylbutazon (bis zu 13,4 mg/kg KG/Tag) über einen Zeitraum von 76 Tagen hinweg erhalten hatte.

Der Befund, wonach die hauptsächlich von den durch Phenylbutazon verursachten Läsionen betroffene Körperstelle beim Pferd der Dickdarm ist, mag erstaunlich erscheinen. Denn bekanntlich neigen die NSAIDs dazu, bei anderen Spezies Schädigungen im Duodenum, im Magen und im Dünndarm zu verursachen. In der Tat wäre zu erwarten, daß der obere Verdauungstrakt im stärksten Ausmaß betroffen ist, weil die genannten Abschnitte nach oraler Verabreichung den höchsten Konzentrationen der Substanz ausgesetzt sind. Möglicherweise ist die offenkundige Abweichung mit der Adsorption eines Großteils der applizierten Stoffmenge von Phenylbutazon an die Faser- und Zellulosebestandteile von Heu und anderem Rauhfutter zu erklären. Es ist denkbar, daß das Arzneimittel dann erst nach der Fermentation in den kaudalen Teilen des Verdauungstrakts freigesetzt wird.

Mit dieser Hypothese ließen sich die von Phenylbutazon im Dickdarm hervorgerufenem Läsionen begründen und auch die deutlich betonte Verzögerung, die bei heugefütterten Ponies bei der Absorption des Wirkstoffs ins Plasma auftritt (Lees, Maitho, Millar und Taylor, 1982, 1983 b). Durch Futterentzug für einige Stunden vor und nach der Verabreichung von Phenylbutazon an Pferde könnte möglicherweise eine rasche Absorption erzielt und ein wirksamer Spiegel der Substanz im Organismus erreicht werden. Ebenso bliebe damit die Schädigung des Dickdarms minimal. Andererseits verlagert die Applikation von Phenylbutazon auf leeren Magen vielleicht lediglich den Ort, an dem es zu Läsionen kommt, zum Magen und Dünndarm hin. Wenn außerdem beim Pferd wie bei einigen anderen Tierarten große Mengen Phenylbutazon mit der Gallenflüssigkeit sekretiert werden, dann gelangt das Medikament immer noch in hoher Konzentration in den Dickdarm. Die biliäre Exkretion stellt wirklich eine plausible Erklärung für die Caecumruptur und den Tod nach parenteraler Phenylbutazon-Verabreichung an Pferde dar, von denen MacKay, French, Nguyen und Maybew (1983) berichteten.
Junge Ratten erwiesen sich als weniger empfindlich gegenüber den Reizwirkungen der NSAIDs im Magen, verglichen mit erwachsenen Tieren (Wilhelmi und Mendasse-Gydnia, 1972). Nach der Phenylbutazon-Applikation bei 3 bis 10 Monate alten Fohlen $(10 \mathrm{mg} / \mathrm{kg} \mathrm{KG}$ täglich 12 bis $42 \mathrm{Ta}$ ge lang) zeigten 9 von 10 Tieren keine klinischen Erscheinungen. Dagegen erbrachten die endoskopische Untersuchung und die Röntgenuntersuchung der lebenden Tiere sowie die spätere Sektion Ulzerationen in großer Häufigkeit (Traub et al., 1983 a). In Anbetracht der Erfahrungen der Autoren mit der Phenylbutazon-Anwendung bei erwachsenen Pferden (Lees et al., 1983 a) erscheint es eigentlich lohnend, den Faktor Alter bei der Empfindlichkeit gegenüber den toxischen Wirkungen des Arzneimittels zu untersuchen. Snow et al. (1981 b) kamen zu dem Schluß, daß die Tiere der verschiedenen Pferderassen unterschiedlich sensibel auf die toxischen Effekte von Phenylbutazon reagieren und daß Ponyrassen empfindlicher als Vollblüter sind. Die fehlenden klinischen Anzeichen von Toxizität bei Vollblütern können aber die Folge der verabreichten niedrigeren Dosen sein $(8,2 \mathrm{mg} / \mathrm{kg} \mathrm{KG}$ pro Tag in zwei Gaben 13 Tage lang, verglichen mit $12 \mathrm{mg} / \mathrm{kg} \mathrm{KG}$ täglich, die Ponies, verteilt auf zwei gleich große Dosen, 8 bis 10 Tage lang erhielten).

Vor einiger Zeit gab man 5 ausgewachsenen Vollblütern und Huntern 15 Tage lang Phenylbutazon (4 Tage lang 4,4 $\mathrm{mg} / \mathrm{kg} \mathrm{KG}$ zweimal täglich; darauf 4 Tage lang $2,2 \mathrm{mg} / \mathrm{kg}$ KG zweimal täglich; dann 7 Tage lang 2,2 $\mathrm{mg} / \mathrm{kg} \mathrm{KG}$ einmal täglich). Es war eine Herabsetzung der Plasmaproteinspiegel um durchschnittlich 16 Prozent festzustellen (Lees et al., 1983 a). In einer zweiten Untersuchung betrug die erste Applikationsphase statt 4 Tagen mit $4,4 \mathrm{mg} / \mathrm{kg} \mathrm{KG}$ zweimal täglich nur noch einen Tag. Keine klinischen Erscheinungen von Toxizität oder toxikologischen Veränderungen im Plasma und in der chemischen Blutzusammensetzung traten auf (Taylor et al., 1983). Diese Befunde stehen in Übereinstimmung mit Klinikberichten von Pferden, die niedrige Erhaltungsdosen von Phenylbutazon über lange Zeiträume hinweg erhielten. Diese lagen unterhalb der Toxizitätsgrenze. Wohl bekannt ist in diesem Zusammenhang, daß die Pferde unter Umständen ein Futter mit darin enthaltener erhöhter Phenylbutazonmenge mehrere Tage lang verweigern. Darin könnte sich eine die Aufnahme betreffende Autoregulation äußern, die toxische Wirkungen verhütet.

Gelegentlich treten auch andere toxische PhenylbutazonEffekte bei Pferden auf. Oft waren dann aber die verabreichten Dosen höher als die vom Hersteller empfohlenen. Daher haben die Befunde nicht immer Bedeutung für die klinische Anwendung. Alexander (1982) gab Shetland-Ponies 4 Tage lang fast $7,5 \mathrm{mg} / \mathrm{kg} \mathrm{KG}$ täglich und berichtete über eine leichte Azidose und eine Abnahme der Natriumund Chloridkonzentration im Harn und der Chlorid-Exkretion mit den Faeces. Diese Veränderungen ähneln den Erscheinungen der Salzretention, die oft beim Menschen in frühen Stadien der Phenylbutazon-Therapie zu beobachten sind. Wahrscheinlich spiegeln sie die aldosteronähnlichen Eigenschaften des Medikaments wider. 
In einer retrospektiven Arbeit über 16 Pferde beschrieb Gunson (1983) eine beidseitige Nekrose der Nierenpapillen nach NSAID-Behandlung. Von 15 Pferden erhielten 11 Phenylbutazon, einigen Tieren wurden aber auch noch andere NSAIDs (gewöhnlich Flunixin) verabreicht. Ebenso kamen in manchen Fällen nephrotoxische Antibiotika wie Gentamycin und Kanamycin zur Anwendung. Die NSAIDs brauchen also nicht unbedingt allein für die beobachteten toxischen Symptome verantwortlich zu sein. Es liegen aber Berichte über Nierenpapillennekrose bei anderen Spezies einschließlich des Menschen in der Folge von NSAID-Applikation vor. Daher waren sie wahrscheinlich doch beteiligt. Gunson (1983) brachte die NSAID-Therapie bei der Entstehung von Nekrosen mit der Dehydratation in Verbindung.

In der darauffolgenden Zeit führten dann Gunson und Soma (1983) den Beweis, daß weder eine Phenylbutazon-Therapie $(8,8 \mathrm{mg} / \mathrm{kg} \mathrm{KG/Tag})$ noch Wasserentzug für ein bis zwei Tage Nierenpapillennekrosen verursachen, sondern nur beide Faktoren gemeinsam. Diese Autoren erstellten die Hypothese, daß sich ihre Befunde einer Hemmung der renalen Prostaglandinsynthese zuschreiben ließen. Die Prostaglandine spielen vermutlich physiologischerweise eine Rolle als Vasodilatatoren bei hyppovolämischen Zuständen zur Erhaltung des Blutflusses im Nierenmark. Bei normovolämischen Tieren üben sie diese Funktion dagegen nicht aus. Read (1983) erhob in seinem Bericht etwa dieselben Befunde.

Extreme Depression, Anorexie und Fieber erschienen in Beschreibungen von Pferden nach Erhalt einer hohen intravenös (iv) verabreichten Phenylbutazon-Dosis (Wanner, Rollinghoff, Gerber und Preisig, 1979). Es bestand der Verdacht auf Hepatotoxizität. Die Untersuchungen der genannten Autoren ließen bei einem Pferd, dem man 4 Tage lang eine tägliche Dosis von $8,8 \mathrm{mg} / \mathrm{kg} \mathrm{KG}$ gab, einen Anstieg der Plasma-Leberenzymspiegel erkennen (Lees et al., 1983 a). Bei halbierter täglicher Applikationsmenge kehrten die Werte in den Normalbereich zurück. Dies läßt eine direkte Beziehung zwischen Dosierung und Zellschädigung des Leberparenchyms vermuten. Wahrscheinlich ist die hepatische Biotransformation der Hauptfaktor in der Bestimmung der Phenylbutazon-Halbwertszeit beim Pferd. Die Autoren warnten deshalb auf der Grundlage, daß eine Leberfunktionsstörung möglicherweise zu einem Circulus vitiosus von Kumulation und noch stärkerer Toxizität führt, vor der Verabreichung hoher Dosen.

Einige Beweise liegen dafür vor, daß Phenylbutazon beim Pferd die zellulären Blutbestandteile beeinflußt, und es existieren viele Berichte über Störungen der Blutzusammensetzung beim Menschen. Wanner et al. (1979) beschrieben eine Leukopenie mit nachfolgender Leukophilie nach iv Applikation hoher Phenylbutazon-Dosen. Die Autoren machten Ausführungen über ähnliche Reaktionen nach oraler Anwendung (Lees et al., 1983a). Auch Snow und Douglas (1983) gaben einen Abfall der Anzahl neutrophiler Zellen und einen zahlenmäßigen Anstieg der Lymphozyten an. Meschter, Maylin und Krook (1984) injizierten im Rahmen einer neueren Untersuchung 3 ausgewachsenen Vollblut-Wallachen Phenylbutazon-Dosen von $13,63 \mathrm{mg} /$ kg KG (dreifache therapeutische Dosierung) iv an 3 aufeinanderfolgenden Tagen. Ein Pferd erhielt 4 Tage später noch eine vierte Gabe. Bei allen Tieren traten Degeneration und Dilatation der Wände der kleinen Venen auf. Die Venenveränderungen galten als erster Schritt bei der Verursachung anderer toxischer Manifestationserscheinungen, darunter Leuko- und Erythrodiapedese, Ulzerationen der Schleimhaut des Magen-Darm-Trakts sowie eine deutlich veränderte biochemische Zusammensetzung des Serums und ebensolche Blutwerte.

Es fand eine Beweisführung zugunsten der These statt, daß die Darreichungsform von Phenylbutazon vielleicht ein Faktor bei der Bestimmung des Grades der Wirkstofftoxizität ist. Snow und Douglas (1983) vertraten die Auffassung, daß Phenylbutazon als Paste toxischer für Ponies ist als in Pulverform. Die Injektionspräparate des Arzneimittels haben starke Reizwirkung, was vor allem auf die hohe Konzentration des Stoffs in Lösungen zurückzuführen und eine Folge des alkalischen pH-Werts ist. Eine perivaskuläre Injektion kann schwere Schäden bei Pferden nach sich ziehhen. Ungeachtet der Applikationsart und des Applikationswegs steht jedoch heute bei Berücksichtigung aller Erkenntnisse fest, daß der Grad der toxischen Phenylbutazon-Wirkung in allererster Linie von der Dosierung abhängt. Wahrscheinlich spielen auch Alters- und Rasseunterschiede eine Rolle, was die Empfindlichkeit angeht, ebenso die Darreichungsform. Bis heute gibt es dafür jedoch keine eindeutigen Belege.

\section{Meclofensäure}

Die vorhandene klinische Erfahrung in bezug auf andere NSAIDs neben Phenylbutazon ist begrenzter und die Zahl der durchgeführten experimentellen Untersuchungen kleiner. Trotz geringerer Kenntnisse ihre toxischen Wirkungen bei Pferden betreffend lassen die ähnlichen Wirkungsweisen und toxischen Effekte aus Berichten von anderen Spezies Vorsicht auch im Umgang mit diesen Arzneimitteln erforderlich erscheinen. Es ist ratsam, die Empfehlungen der Hersteller hinsichtlich Dosierung zu befolgen. Die Autoren geben beispielsweise einen mittleren Abfall der Plasma-Proteinkonzentration von 18 Prozent bei Ponies an, die 10 Tage lang die empfohlene Dosis von Meclofensäure $(2,2 \mathrm{mg} / \mathrm{kg} \mathrm{KG}$ einmal täglich) erhielten ( $P$. Lees und W. E. Allen; unveröffentlichte Meßwerte). Snow et al. (1983) beschrieben ähnliche Beobachtungen, obgleich in keiner der beiden Untersuchungen klinisch Anzeichen einer Vergiftung auftraten. Snow äußerte die Vermutung, daß eine meclofensäureinduzierte Hypoproteinämie unter Umständen nicht von einer zum Proteinverlust führenden Darmerkrankung herrührt. Zu den anderen möglichen Ursachen gehören herabgesetzte Futteraufnahme, verminderte Eiweißsynthese in der Leber und erhöhte renale Ausscheidung. Zur Bestätigung dieser Verdachtsgründe bedarf es experimenteller Studien.

Die Verabreichung von $2,2 \mathrm{mg} / \mathrm{kg} \mathrm{KG}$ Meclofensäure täglich (entsprechend der empfohlenen Dosierung) an Hengste über 84 aufeinanderfolgende Tage hinweg und an Stuten an 152 aufeinanderfolgenden Tagen, darin die 130 ersten Tage der Gravidität, zogen keinerlei toxische Erschei- 
nungen nach sich (G. A. Smith, 1980; persönliche Mitteilung). Bei den Hengsten war die Libido unvermindert, und es traten keine Veränderungen an den Spermatozoen auf; die Stuten blieben gesund und brachten normale Fohlen zur Welt. 4 Ponystuten, die in der zweiten Hälfte der Trächtigkeit während zweier 10tägiger Perioden Meclofensäure $(2,2 \mathrm{mg} / \mathrm{kg} \mathrm{KG})$ erhielten, blieben ebenfalls gesund und hatten normale Fohlen, aber die Dauer der Gravidität war gegenüber einer Gruppe von 5 unbehandelten Ponies etwas verlängert (W. E. Allen und $P$. Lees, 1982; unveröffentlichte Angaben). Diese Beobachtung ist möglicherweise ohne Bedeutung, und zur Erhärtung der Befunde sind weitergehende Untersuchungen erforderlich.

Parke Davis liefert auf seinem Beipackzettel zu Arquel Informationen zur Meclofensäure-Toxiżität im Falle einer Applikation sehr hoher Dosen über längere Zeiträume hinweg. $\mathrm{Zu}$ den Symptomen gehören Anorexie, Depression, Schwäche, Konditionsverlust, Ulzerationen an der Backenschleimhaut, Durchfall, okkultes Blut in den Faeces und herabgesetzter Hämatokrit. Die meisten dieser Symptome treten laut Berichten auch nach Phenylbutazon-Überdosierung auf. Bei gleichzeitigem hochgradigem Wurmbefall waren auch leichte Kolik und eine veränderte Konsistenz der Faeces bei Pferden zu beobachten, die unter Meclofensäure-Behandlung standen.

\section{Flunixin}

Die Beschreibung eines Falls von Flunixin-Toxizität liegt vor (Trillo, Soto und Gunson, 1984), in dem eine Pony-Stute 5 Tage lang zweimal täglich iv eine Dosis von $3 \mathrm{mg} / \mathrm{kg} \mathrm{KG}$ erhielt. Diese Menge ist mehr als die fünffache empfohlene Dosis und führte zu Anorexie, Depression, oraler Ulcusbildung und verschiedenen biochemischen Veränderungen, unter anderem Hypoproteinämie und Neutropenie. Bei der Sektion fanden sich Ulzerationen in Magen und Colon. Diese Befunde weisen darauf hin, daß die iv Applikation von Flunixin toxische Erscheinungen im Magen-DarmTrakt zur Folge haben kann, aber die sehr hohe Dosierung läßt Zweifel an ihrer klinischen Relevanz aufkommen. Hinzu kommt, daß bei anderen Untersuchungen klinisch und biochemisch keine Anzeichen von Toxizität nach Verabreichung einer drei- bzw. fünffachen iv Dosis über 10 bzw. 5 Tage hinweg vorhanden waren (Houdeshell und Hennessey, 1977).

Auch eine 15tägige orale Applikation der dreifachen empfohlenen oder derselben Menge, an 10 aufeinanderfolgenden Tagen intramuskulär injiziert, konnte keine toxischen Wirkungen hervorrufen. Das Medikament scheint also hinsichtlich Dosierung einen ausreichenden Sicherheitsspielraum zu besitzen. Trotzdem empfahlen Webbon und Woolliscroft (1984), bei der Anwendung über lange Zeiträume

\section{Das starke Antiphlogistikum für Pferde:}

\section{Apirel ${ }^{\circledR}$}

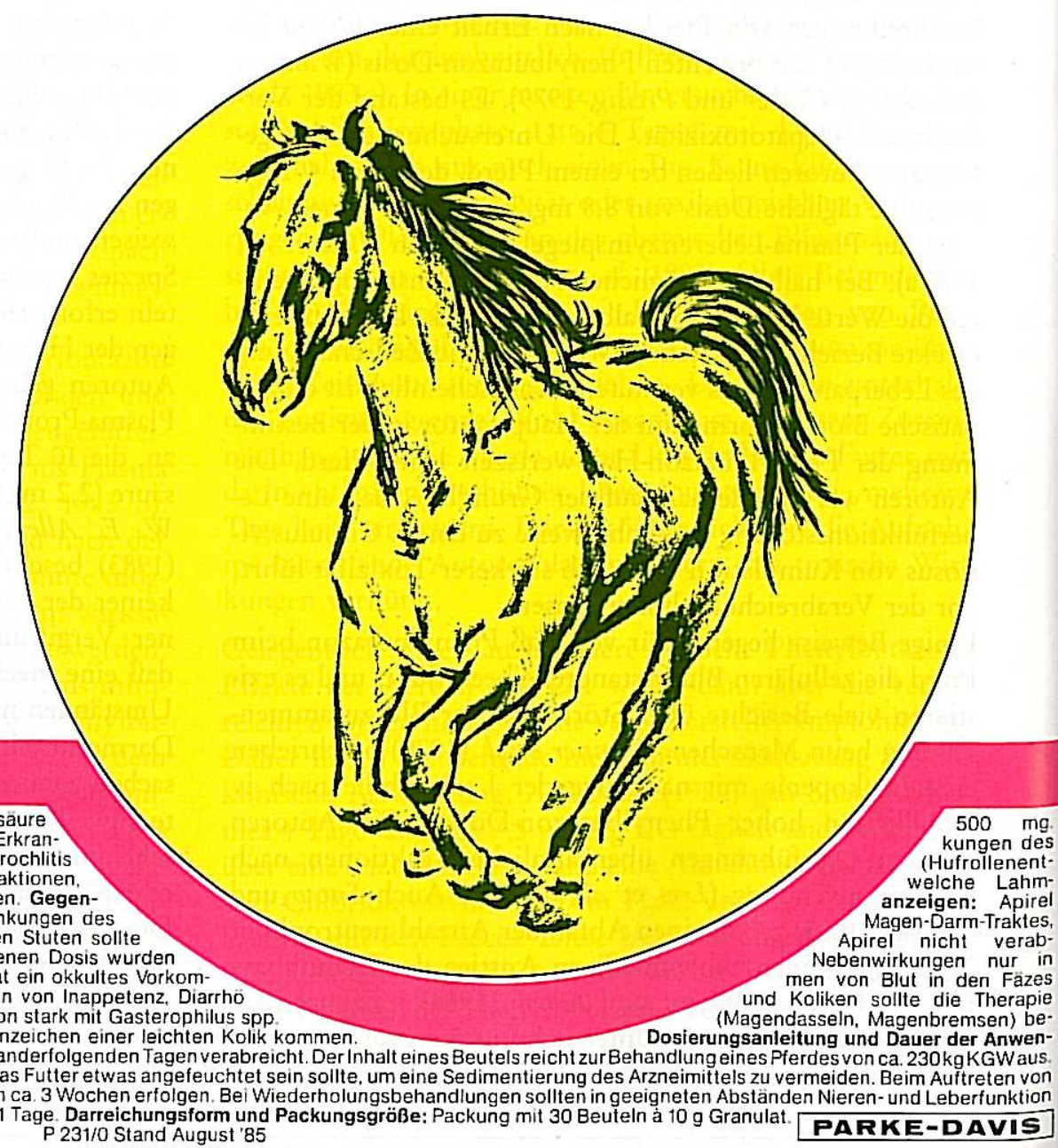

Zusammensetzung: $10 \mathrm{~g}$ Granulat enthalten: Meclofenaminsäure Anwendungsgebiete: Alle akuten und chronischen entzündlichen ErkranBewegungsapparates beim Pferd, wie z.B. Osteoarthritis, Podotrochlitis zundung), Hufrehe, Bursitis, Tendinitis, Osteitis; entzundliche Reaktionen, hollte nicht eingesetzt werden bei Pferden, z.B. nach Verletzungen. Gegender Leber, der Nieren oder des blutbildenden Systems; der Leber, der Nieren oder des blutbildenden Systems; trächtigen Stuten solite reicht werden. Nebenwirkungen: Bei Verabreichung der empfohlenen Dosis wurden ganz vereinzelten Fallen beobachtet. Bei hoheren Dosierungen trat ein okkultes Vorkom-
und ein Absinken des Hamatokritwertes auf. Bei ersten Anzeichen von Inappetenz. Diarrho abgebrochen werden. Bei Pferden, die zum Zeitpunkt der Medikation stark mit Gasterophilus spp fallen sind, kann es zu einer Konsistenzanderung der Fazes und Anzeichen einer leichten Kolik kommen.

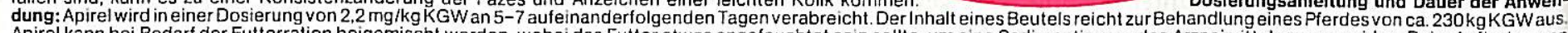

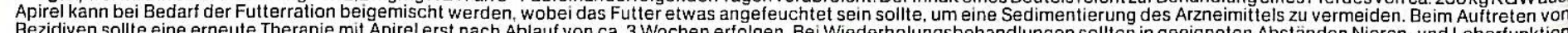

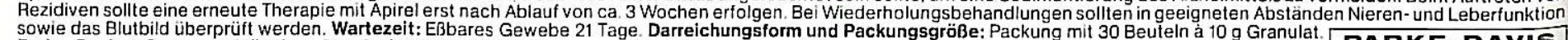
Parke, Davis \& Company, München, Postfach 5620, 7800 Freiburg $\quad$ P 231/0 Stand August ' 85 
vorsichtig zu sein. Sie beschrieben einen Fall von Hypoproteinämie und Hypalbuminämie bei einem Shetland-Pony nach Flunixin-Behandlung. Gelegentlich trat an der Stelle der intramuskulären Injektion eine leichte Schwellung auf, wohingegen über eine Thrombophlebitis nichts bekannt ist.

\section{Naproxen}

Naproxen hat, wie es ausgehend von den verfügbaren Meßwerten scheint, wie Flunixin beim Pferd einen ausreichenden Sicherheitsspielraum, was die Dosierung betrifft. Bei einer 42tägigen (dreimal so lange wie der angeratene Zeitraum) oralen oder iv Anwendung der dreifachen empfohlenen Dosis traten keine Toxizitätserscheinungen auf (Tobin, 1979 b). Darüber hinaus berichteten Snow et al. (1983), daß die Verabreichung von Naproxen 14 Tage lang in der vorgesehenen Menge, gefolgt von doppelt so hohen Gaben für 7 weitere Tage, die Plasma-Proteinkonzentration nicht negativ beeinflußte.

\section{Andere NSAIDs}

Roberts (1982) beschrieb inverse Reaktionen auf Indomethacin beim Pferd. Einzelgaben von Mengen zwischen 3 und $9 \mathrm{mg} / \mathrm{kg} \mathrm{KG}$, oral appliziert, führten zu Teilnahmslosigkeit, Desorientiertheit, Lähmungen, Neutropenie und okkultem Blut in den Faeces. Andere Versuche am Pferd, bei denen Indomethacin in Dosen von etwa $2,5 \mathrm{mg} / \mathrm{kg} \mathrm{KG}$ täglich angewandt wurde, verursachten klinisch keine Anzeichen von Toxizität (Hunt, Haywood und Moss, 1979; Phillips, Salyer und Ray, 1980). Die Allgemeingültigkeit von Roberts' Befunden steht daher nicht fest, besonders weil seine Dosierung über der zur Erzielung einer therapeutischen Wirkung erforderlichen gelegen haben mag. Letztere hat man bisher nicht ermittelt, und Indomethacin ist sowieso derzeit nicht unter Angabe von Indikationen für den Gebrauch beim Pferd im Handel.

Judson und Barton (1981) haben eine Verlängerung der Blutungszeit und eine verminderte Klebrigkeit der Thrombozyten nach einer einzigen oralen Gabe von Aspirin (20 $\mathrm{mg} / \mathrm{kg} \mathrm{KG}$ ) beim Pferd nachgewiesen. Diesen Effekten liegt, so lautete eine Vermutung, eine reduzierte Bildung von $\mathrm{TXA}_{2}$, einem wirkungsvollen proaggregatorischen Stoff in den Blutplättchen, zugrunde. Nach Ansicht der Autoren könnte dies die Basis für einen klinisch nutzbaren antithrombotischen Effekt der NSAIDs zum Beispiel bei Hufrollenerkrankung sein. Andererseits soll es auch als eine mögliche Ursache der Epistaxis in Betracht kommen. Sie tritt häufig bei Pferden im Training während oder nach einer körperlichen Anstrengung auf (Trujillo, Rios, Maldonado und Rudolph, 1981). Trujillo und seine Kollegen verabreichten Pferden oral in jeder zweiten Woche an fünf Wochentagen 6 oder $12 \mathrm{~g}$ Aspirin. Darauf war im Vergleich zu Kontrolltieren die Thrombozytenzahl erniedrigt und Gerinnungs-, Prothrombin- und Blutungszeit waren erhöht. Ausgehend von diesen Befunden könnte die Anwendung von Aspirin über längere Zeiträume hinweg hämorrhagische Probleme verursachen oder in dieser Richtung prädisponierend wirken. Berichte über ähnliche Untersuchungen mit häufig applizierten NSAIDs liegen nicht vor. Bei In-vitro-Versuchen mit Thrombozyten vom Pferd ließen sich aggregationshemmende Eigenschaften von Naproxen, Phenylbutazon und Flunixin nachweisen, wobei Flunixin am wirkungsvollsten war (Johnstone, 1983). Über die Relevanz solcher Untersuchungen für den klinischen Gebrauch der Stoffe ist nichts bekannt.

\section{Pharmakokinetik}

\section{Allgemeine Überlegungen}

Ein sehr großer Teil der NSAID-Plasmakonzentration, die man mit klinischen Dosierungen erzielt, ist normalerweise an das Plasmaprotein gebunden. Wie Tabelle 3 zeigt, liegen unter Umständen über 99 Prozent der Gesamtmenge im Plasma in proteingebundener Form vor. Wahrscheinlich ist dieser Tatbestand für den relativ geringen Anteil der meisten NSAIDs im Speichel und denjenigen Teil verantwortlich, der unverändert mit dem Harn ausgeschieden wird. Mit dem hohen Proteinbindungsvermögen läßt sich auch erklären, weshalb geringere Konzentrationen von Phenylbutazon und Naproxen in der Synovialflüssigkeit des Pferds zu finden sind, verglichen mit Serumspiegelwerten (Lebmann, Wintzer und Frey, 1981). Dagegen sind die Wirkstoffkonzentrationen in entzündeten Gelenken erwartungsgemäß wohl höher als in normaler Synovialflüssigkeit. Das hat seinen Grund im vermehrten Blutfluß, in erhöhter Gefäßdurchlässigkeit und Penetration von Proteinen hin zu entzündlich veränderten Stellen (Higgins und Lees, 1984).

Die Wirksamkeit der NSAIDs hängt wahrscheinlich wesentlich von der Verstoffwechselung in der Leber des Pferds ab, für die meisten Arzneimittel stehen in dieser Hinsicht jedoch keine detaillierten Untersuchungsergebnisse zur Verfügung. Die Ausscheidung der NSAIDs auf nichtrenalen Stoffwechselwegen könnte sich ebenfalls als besonders interessant erweisen. Bei einigen Spezies werden beträchtliche Stoffmengen mit der Galle sekretiert. Am Pferd hat man diesen exkretorischen Stoffwechselweg, ausgenommen beim Phenylbutazon, nicht untersucht. Möglicherweise kann aber dieser pharmakokinetische Weg die Ulzerogenität eines NSAIDs steigern, indem er hohe Konzentrationen im Dünn- und Dickdarm auftreten läßt.

\section{Phenylbutazon}

Phenylbutazon zeigt beim Pferd ein dosisabhängiges pharmakokinetisches Verhalten. Mit zunehmender Dosierungshöhe nimmt die Plasma-Halbwertszeit von 3 auf $8 \mathrm{~h} \mathrm{zu}$ (Tabelle 4) (Piperno et al., 1968; Lebmann et al., 1981). Piperno und seine Mitarbeiter zeigten deutlich den Effekt der Größe der iv Injektionsmenge auf die Plasmakonzentration. Durch Verdoppelung der Phenylbutazon-Dosis von 4,4 auf $8,8 \mathrm{mg} / \mathrm{kg} \mathrm{KG}$ ließ sich die nach $1 \mathrm{~h}$ gemessene Plasmakonzentration um 33 Prozent steigern. Wenn sich die Dosis gar auf $17,6 \mathrm{mg} / \mathrm{kg} \mathrm{KG}$ erhöhte, nahm der Plasmaspiegel um 300 Prozent zu. Auch noch $9 \mathrm{~h}$ nach der Applikation der kleinen bzw. großen Gabe betrug die Plasmakonzentration 3 bzw. $30 \mu \mathrm{g} / \mathrm{ml}$. Diese Untersuchungen machen die Kumulationsfähigkeit im Plasma und damit die Toxizität bei der Verabreichung hoher Dosen deutlich. Die 


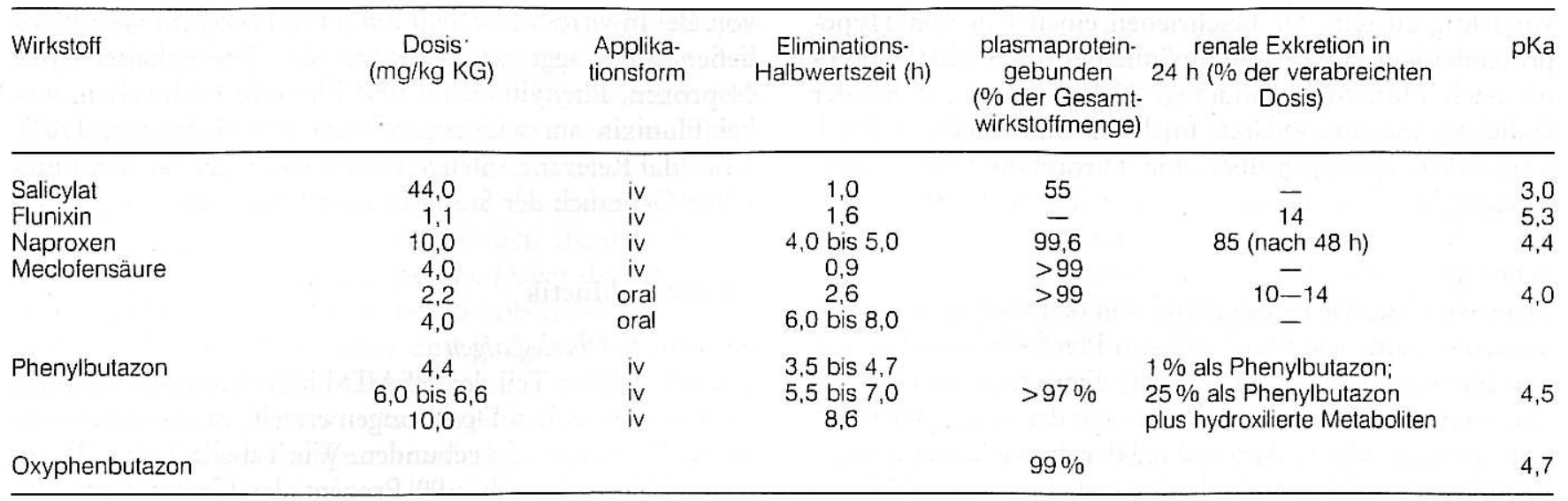

Tab. 3: Pharmakokinetik der NSAIDs beim Pferd

Arbeit von Tobin, Blake und Valentine (1977) liefert eine mögliche Erklärung für diese Befunde. Ihrem Bericht zufolge war die Phenylbutazon-Halbwertszeit bei mit Oxyphenbutazon vorbehandelten Tieren leicht erhöht $(7,6$ bis $9,3 \mathrm{~h})$. Oxyphenbutazon ist ein normaler Metabolit von Phenylbutazon (Maylin, 1974; Gerring et al., 1981). Deshalb könnte es hemmend auf die weitere Verstoffwechselung seiner Vorläuferverbindung in der Leber wirken und dadurch deren Halbwertszeit verlängern. Ein solcher Effekt ließ sich für Oxyphenbutazon bei der Ratte nachweisen (Janchen und Levy, 1972). Die Dosisabhängigkeit der Phenylbutazon-Halbwertszeit kann jedoch auch einfach das Ergebnis einer Sättigung der mikrosomalen Enzyme mit dem eigentlichen Wirkstoff sein.

Moss (1972) wies eine höchst unterschiedliche Absorptionsrate des Phenylbutazons nach oraler Anwendung eines Pulverpräparats nach, und Gerring et al. (1981), Rose, Kobnke und Baggot (1982) sowie Snow und Douglas (1983) bestätigen dies. Unterschiede in der Absorption können bei einem einzelnen Tier auftreten, und sogar dann, wenn alle Voraussetzungen für regelmäßige Futteraufnahme und Konstanthaltung der Versuchsbedingungen erfüllt sind. Rose und seine Kollegen haben darauf hingewiesen, daß die bei Pferden untereinander stark differierenden Phenylbutazon-Plasmaspiegel eventuell der Grund für eine erfolglose
Therapie sind. Andere haben jetzt gezeigt, daß geringere individuelle Unterschiede auftreten, wenn der Patient vor der Medikation kein Futter erhalten hat (Maitho, Lees und Taylor, 1985).

Mehrere Forscher haben sich in experimentellen Arbeiten am Pferd speziell damit befaßt, den Einfluß der Fütterung auf die Absorptionsrate von Phenylbutazon und auf die absorbierte Gesamtmenge zu bestimmen. Rose et al. (1982) verabreichten in einer einzigen Gabe oral die doppelte Menge der normalerweise für diese Applikationsform empfohlenen Dosis, und zwar einmal in einer kleinen Menge Kleie-Mash und einmal nach einer vollen Mahlzeit. Die Zeitspanne bis zum Erreichen der höchsten Konzentration war vergrößert, und die Fläche unter der Plasmakonzentration-Zeit-Kurve (AUC), anhand deren sich die absorbierte Gesamtmenge abschätzen läßt, war unter „satten“ Bedingungen verkleinert. Sullivan und Snow (1982) beschrieben eine mit etwa derselben Verzögerung erreichte Plasma-Spitzenkonzentration von Phenylbutazon und eine stark verkleinerte AUC. Sie hatten Phenylbutazon nach einer Mahlzeit im Rahmen des täglichen Fütterungsplans gegeben. Die Auswirkungen traten bei erwachsenen Vollblütern ebenso wie bei Fohlen auf.

In beiden Untersuchungen bestimmte man die AUC nur in der Zeit zwischen 0 und $12 \mathrm{~h}$, und so war die Feststellung

Tab. 4: Plasmakonzentration: Konzentration im entzündlichen Exsudat - Verhältnis entzündungshemmender Arzneimittel beim Pferd

\begin{tabular}{|c|c|c|c|}
\hline Zeit nach der Injektion & Phenylbutazon ${ }_{\mathrm{P}}^{*}$ & Oxyphenbutazon ${ }_{\mathrm{P}}^{*}$ & Flunixin $P_{P}^{+}$ \\
\hline (h) & Phenylbutazon $_{E}$ & Oxyphenbutazon $_{E}$ & Flunixin $_{E}$ \\
\hline 3 & - & - & $1,88(6)$ \\
\hline 6 & $1,64(7)$ & $1,07(7)$ & $0,56(6)$ \\
\hline 12 & $0,56(7)$ & $0,55(7)$ & $0,21(4)$ \\
\hline 24 & $0,33(6)$ & $0,43(6)$ & - \\
\hline
\end{tabular}

\section{P Plasma}

$E$ entzündliches Exsudat

* Plasmakonzentration: Konzentration im entzündlichen Exsudat - Verhältnis nach iv Injektion von Phenylbutazon (4,4 mg/kg KG) (nach Higgins et al., 1984)

† Plasmakonzentration: Konzentration im entzündlichen Exsudat - Verhältnis nach iv Injektion von Flunixin (1,1 mg/kg KG) (nach Lees und Higgins, 1984)

Die Werte in Klammern geben die Zahl der Tiere an. 
einer ausgeprägten Zunahme der zur Erreichung der höchsten Plasmakonzentration benötigten Zeit bei 6 Ponies von Interesse, die Zugang zu Heu hatten (Lees et al., 1982, 1983 b). Spitzenkonzentrationen wurden durchschnittlich nach 13,2 h gemessen. Bei einzelnen Ponies stellten sie sich 15 und manchmal sogar $18 \mathrm{~h}$ nach einer oralen Dosis von $4,4 \mathrm{mg} / \mathrm{kg} \mathrm{KG}$ ein. Im Gegensatz zu den Befunden von Rose et al. (1982) und Sullivan und Snow (1982) war weder die AUC deutlich verkleinert noch die Plasma-Spitzenkonzentration markant erniedrigt. Der einzige Effekt der Heufütterung war die verzögerte Absorption, ohne daß dabei die zum Schluß absorbierte Gesamtmenge geringer war. Wenn der Grund hierfür die Adsorption des Wirkstoffs gerade an die Rohfaser ist, dann ließen sich ihr die vorgefundenen ulzerierten Stellen im kaudalen Darmabschnitt (siehe oben) zuschreiben. Ebenso würde sie aber auch vergrößerte Zeitspannen vor Eintritt der therapeutischen Wirkung nach einer oralen Phenylbutazon-Applikation erklären. Dies hat besonders für Pferde Bedeutung, die an Wettkämpfen unter International Equestrian Federation Regulations teilnehmen. Sie lassen derzeit einen Höchstwert von $4 \mu$ g Phenylbutazon/ml Plasma zu. Möglicherweise ist mit dieser Hypothese auch zu begründen, daß bei zwei Ponies nach oraler Verabreichung überhaupt kein Phenylbutazon im Plasma nachweisbar war. Diese Tiere hatten nämlich eine halbe Stunde vor der Medikation Heu bekommen (Snow und 'Douglas, 1983).

Vielleicht hat auch die Tageszeit einen Einfluß auf die Geschwindigkeit und Menge der Phenylbutazon-Absorption nach oralen Gaben. In Arbeiten der Autoren (Lees et al., 1983 b) lauten aber die Angaben, die Bioverfügbarkeit betreffend, auf 72 bzw. 71 Prozent, wenn die Arzneimittelapplikation um $11.00 \mathrm{~h}$ bzw. $16.00 \mathrm{~h}$ erfolgte. Allerdings war die Absorptionsgeschwindigkeit morgens höher als nachmittags. Rasseunterschiede können bei der Absorption ebenfalls eine Rolle spielen; Sullivan und Snow (1982) berichteten über etwas höhere AUC-Werte bei Vollblütern als bei Ponies, aber aus unveröffentlichten Studien $(P$. Lees und J. B. Taylor, 1982) geht das Gegenteil hervor.

Mit dem Harn werden beim Pferd nur geringe Mengen Phenylbutazon ausgeschieden. Bei einer Untersuchung betrug der in $24 \mathrm{~h}$ exkretierte Anteil der oral oder iv applizierten Dosis 2 Prozent (Lees et al., 1983 b). Dies hängt wahrscheinlich damit zusammen, daß ein großes Plasmabindungsvermögen nur wenig davon im Glomerulärfiltrat erscheinen läßt. Biliäre Sekretion mit oder ohne nachfolgende Reabsorption tritt bei Pferden wohl auf. Zu dieser Vermutung führte die Beobachtung, daß über einen Zeitraum von $70 \mathrm{~h}$ hinweg 29 Prozent einer iv und 21 Prozent einer oralen Dosis Phenylbutazon in den Faeces erschienen (P. B. W. Smith, J. Caldwell, R. L. Smith, H. W. Horner, E. Houghton und M. S. Moss, 1984; persönliche Mitteilung). Bekanntermaßen wird ein beträchtlicher Anteil jeder verabreichten Phenylbutazon-Dosis im Stoffwechsel hydroxyliert, vornehmlich in der Leber. Maylin (1974) und Gerring et al. (1981) identifizierten zwei Derivate, Oxyphenbutazon (Ring-Hydroxylierung) und Gammahydroxyphenylbutazon (Seitenketten-Hydroxylierung). Gammahydroxyphenylbutazon kommt im Plasma in sehr niedriger Kon- zentration vor, dagegen in hoher Konzentration im Harn (Harn-Plasma-Konzentration-Quotienten von 400 sind nicht ungewöhnlich), was auf eine tubuläre Sekretion in der Niere hinweist. Oxyphenbutazon ist besonders interessant, weil sein Plasmaspiegel 10 bis 25 Prozent eines gleichzeitig vorhandenen Phenylbutazon-Spiegels erreichen kann. Der Metabolit hat entzündungshemmende Eigenschaften und könnte somit zur Gesamt-Körperreaktion beitragen, besonders weil seine Spiegel im entzündlichen Exsudat diejenigen im Plasma übersteigen können (Higgins et al., 1984). Oxyphenbutazon kommt ebenfalls über die tubuläre Sekretion in der Niere zur Exkretion.

Die Autoren untersuchten den Harn männlicher Ponies aus einer 24stündigen Sammelperiode auf die ausgeschiedenen Anteile der verabreichten iv Dosis Phenylbutazon an unverändertem Medikament und seinen Metaboliten. Sie fanden 2 Prozent Phenylbutazon, 12 Prozent Oxyphenbutazon und 11 Prozent Gammahydroxyphenylbutazon insgesamt 25 Prozent (Lees et al., 1983 b). Auch bei Berücksichtigung eines dritten Metaboliten im Pferdeharn, den die Autoren in der nachfolgenden Zeit entdeckten (Gammahydroxyphenylbutazon) (J. B. Taylor und P. Lees, 1982; unveröffentlichte Daten) ist der Verbleib von über 70 Prozent der applizierten Menge unbekannt. Daher gibt es anscheinend wohl noch andere, bisher nicht isolierte Metaboliten von Phenylbutazon. Denkbar wäre auch eine Exkretion des Wirkstoffs und/oder seiner Stoffwechselprodukte auf anderen Wegen als im Harn (wie zum Beispiel mit der Galle). Oder das Medikament und/oder seine Metaboliten binden sich vielleicht an bestimmte Körpergewebe, aus denen es/sie nur langsam freigesetzt wird/werden:

\section{Meclofensäure}

Bei Pferden iv injizierte Meclofensäure hat eine Halbwertszeit von 0,9 h (Snow, Baxter und Whiting, 1981a). Nach oraler Verabreichung findet normalerweise eine rasche $\mathrm{Ab}$ sorption statt, und nach 0,5 bis 4 h stellt sich die Höchstkonzentration ein. Eine Verzögerung kann bei Pferden auftreten, die freien Zugang zu Heu haben (P. Lees und W. E. Allen; unveröffentlichte Meßwerte). Die Clearance läuft ziemlich rasch $a b$, und so findet sich normalerweise $24 \mathrm{~h}$ nach oraler Medikation ein sehr niedriger Plasmaspiegel. Nach der Applikation per os, bei der die Halbwertszeit 2,6 h beträgt, ist die Plasma-Clearance aber etwas langsamer als nach iv Injektion (Snow et al., 1981a). In früheren Arbeiten gaben Forscher etwas höhere Werte für die Halbwertszeit an, 3 bis 4 h (Sams, 1979) bzw. 6 bis 8 h (Conner, Riley, Beck und Coppock, 1973) bei oraler Verabreichung. Snow et al. (1981a) zeigten auch, daß die orale Absorption von Ponies, die gefressen hatten, und solchen, die gehungert hatten, etwa gleich ist.

Detaillierte Ausführungen über den Stoffwechsel von Meclofensäure beim Pferd stehen nicht zur Verfügung. Man weiß nur, daß sie wahrscheinlich mit Glucuronsäure konjugiert wird (P. Lees und W. E. Allen; unveröffentlichte Daten). Die Harnkonzentration ist ungefähr $25 \mathrm{mal}$ so hoch wie der Plasmaspiegel, und etwa 10 bis 14 Prozent der oral gegebenen Menge werden mit dem Harn ausgeschieden ( $G$. A. Smith; persönliche Mitteilung). Unter Umständen findet 
auch eine biliäre Sekretion statt. Meclofensäure ist nun eine der wenigen NSAIDs, für die Geweberückstandsuntersuchungen am Pferd durchgeführt wurden. Die höchsten Konzentrationen treten im Gelenkknorpel, im Fettgewebe des Netzes und in den Haaren auf. Rückstände im Harn sind nach einer 7tägigen Dosisabfolge $96 \mathrm{~h}$ lang nachweisbar.

\section{Flunixin}

Nach iv Injektion beträgt die Plasma-Eliminationshalbwertszeit von Flunixin nur 1,6 h. Chay et al. (1982) legten Meßwerte vor, die als Hinweis auf eine weitere, langsamere Ausscheidungsphase schließen lassen könnten, aber dies steht nicht zweifelsfrei fest. Die Harnkonzentration an freiem und konjugiertem Flunixin ist annähernd $40 \mathrm{mal}$ so hoch wie der Plasmaspiegel, so daß die renale Exkretion einen wesentlichen Teil der Stoffwechselvorgänge darstellt, mit der Hilfe das Arzneimittel den Körper verläßt. Nichtsdestoweniger erschienen in einem 24stündigen Zeitraum nur 14 Prozent der applizierten Menge in auf diesem Weg exkretierter Form (Houdeshell und Hennessey, 1977). Der Verbleib des Rests war offenbar noch nicht feststellbar. Geweberückstandsuntersuchungen zeigten lediglich, daß es nicht zu einer Kumulation im Körper kommt. Bei der Ratte ließ sich eine faekale Exkretion nachweisen, so daß Verluste in die Gallenflüssigkeit oder in andere Sekrete des Magen-Darm-Trakts hinein vermutlich auch beim Pferd auftreten. Über Metaboliten von Flunixin beim Pferd ist bisher nichts bekannt.

Die Absorption erfolgt nach oraler und intramuskulärer (im) Verabreichung rasch, und innerhalb von 30 Minuten sind die höchsten Plasmaspiegel erreicht. Der geringe zeitliche Abstand zwischen Einstellung der Plasmakonzentration und der iv und oralen Applikation leitet hin zur Annahme, daß eine nahezu vollständige Absorption stattfindet. Entsprechend geben Chay et al. (1982) eine Bioverfügbarkeit von fast 80 Prozent an. Die Forscher äußerten die Vermutung, die rasche Absorption könnte ein wichtiger Faktor beim Bestreben sein, das Risiko einer ulzerogenen Wirkung möglichst gering zu halten. Auch könnte sie sich dann als nützlich erweisen, wenn eine NSAID-Therapie bei einem Pferd für nötig befunden wird, das kurz darauf in einem Wettkampf starten soll. Andererseits besteht aber wahrscheinlich ein ebenso enger Zusammenhang zwischen der Ulzerogenität und der Plasmakonzentration wie zwischen ihr und dem Spiegel im Magen-Darm-Trakt. Eine rasche Absorption mit nachfolgender schneller Exkretion läßt die Gefahr eines Nachweises des Wirkstoffs im Plasma geringer werden. Dagegen hält die Wirkung des Flunixins in Form von Blockierung der Eikosan-Synthese (Lees und Higgins, 1984) und von Unterdrückung oder Verdeckung einer eventuellen Lahmheit (Houdeshell und Hennessey, 1977) länger an. Die Tatsache, daß kein Flunixin im Plasma zu isolieren ist, bedeutet daher nicht, daß sein klinischer Effekt nicht doch noch vorhanden ist.

\section{Naproxen}

Dieses Medikament ist nur in der Darreichungsform als Granulat zum oralen Gebrauch bei Pferden im Handel.
Vermischt mit dem Futter oder obendrauf gestreut ist es wohlschmeckend, und offenbar treten keine Probleme hinsichtlich etwaiger Futterverweigerung auf. Die Bioverfügbarkeit nach oraler Medikation beträgt bei Einhaltung der empfohlenen Dosierung den Angaben zufolge 50 Prozent. Die Plasma-Spitzenkonzentrationen stellen sich dabei nach 2 bis 3 h ein (Tobin, 1979b). Naproxen und sein Hauptmetabolit, 2-[6-Hydroxinaphtyl]propionsäure, werden hochkonzentriert mit dem Harn ausgeschieden.

Die Plasmahalbwertszeit von Naproxen liegt beim Pferd im Bereich von 4 bis $5 \mathrm{~h}$ (Tobin, $1979 \mathrm{~b}$; Lebmann et al., 1981), also ähnlich wie bei Phenylbutazon. Tatsächlich gleichen sich die beiden Arzneimittel auch darin, daß bei beiden beträchtliche Speziesunterschiede hinsichtlich der angegebenen Plasma-Eliminationsraten bestehen. Bei Naproxen beträgt die Halbwertszeit 12 bis $15 \mathrm{~h}$ (Mensch) bzw. $74 \mathrm{~h}$ (Hund), bei Phenylbutazon $3 \mathrm{~h}$ (Hund), $40 \mathrm{~h}$ (Rind) bzw. $72 \mathrm{~h}$ (Mensch). Als erster Punkt lautet nun bei zweien von den vier in der Pferdeheilkunde hauptsächlich angewandten NSAIDs mit einer Halbwertszeit von etwa 5 h Phenylbutazon und Naproxen, die Empfehlung auf Verabreichung zweimal täglich. Diejenigen mit kürzerer Halbwertszeit dagegen, Meclofensäure $(0,9 \mathrm{~h})$ und Flunixin $(1,6$ h), sollen einmal täglich gegeben werden. Weiterhin ist ungewiß, ob die Wirkung der NSAIDs in einer reversiblen oder irreversiblen Blockierung des Zyklooxygenase-Stoffwechsels besteht. Und drittens ist das Verhältnis zwischen der Plasma-Wirkstoffkonzentration und der Konzentration an entzündlich veränderten Stellen nicht konstant (Higgins et al., 1984). All dies läßt wohl berechtigte Zweifel an den Empfehlungen zu den NSAIDs hinsichtlich Dosisgrößen und Dosierungsintervallen aufkommen.

\section{Andere Medikamente}

Davis und Westfall (1972) untersuchten die Verstoffwechselung von Natriumsalicylat bei Ponies einer Shetland-Kreuzungsrasse. Bei einer iv Dosierung von $44 \mathrm{mg} / \mathrm{kg}$ KG betrug die Plasmahalbwertszeit $1 \mathrm{~h}$. Die entsprechenden Werte für andere Spezies bewegten sich zwischen $0,78 \mathrm{~h}$ bei der Ziege und 37,6 h bei der Katze. Die rasche Plasma-Clearance von Salicylat bei Ponies war hauptsächlich einer schnellen renalen Exkretion durch glomeruläre Filtration zusätzlich zur tubulären Sekretion des unveränderten Stoffs zuzuschreiben. Als schwache Säure begünstigt der alkalische Pferdeharn noch die Salicylat-Ausscheidung über die Niere. Interessant ist, daß Salicylat ein physiologischer Bestandteil des Pferdeharns ist, wenn auch normalerweise mit sehr niedriger Konzentration (Moss, 1972). Moss hat außerdem nachgewiesen, daß hohe Harnkonzentrationen von Salicylat auftreten, wenn man Wintergrünöl (Methylsalicylat) auf die Haut bringt.

Davis und Westfall (1972) zeigten auch, daß eine orale Absorption von Salicylat bei Ponies stattfindet. Die höchsten Konzentrationen waren nach einer Stunde erreicht, aber der Plasmaspiegel blieb nicht in befriedigendem Maß konstant, besonders deshalb, weil auf die rasche Absorption eine schnelle Exkretion folgte. Ebenso demonstrierten Davis, Westfall und Short (1973), daß die Halbwertszeit beim Fohlen mit zunehmendem Alter abnimmt. Im Alter von $30 \mathrm{Ta}$ - 
gen entspricht der Wert dann dem des erwachsenen Tiers. Es ist jedoch zu beachten, daß die kurze Plasmahalbwertszeit von Salicylat beim Pferd nicht notwendigerweise auch eine kurze Wirkungsdauer zur Folge hat. Wie schon zuvor für Flunixin und Meclofensäure, deren Halbwertszeit beim Pferd ebenfalls kurz ist, bemerkt wurde, können die therapeutischen Effekte $24 \mathrm{~h}$ oder länger anhalten. In Anbetracht der offensichtlich geringen Ulzerogenität von Salicylat (Whittle et al., 1980) ist es unter Umständen lohnend, seinen praktischen Wert in der Pferdeheilkunde neu zu überprüfen. Allerdings setzt seine schwache Wirksamkeit seinem erfolgreichen Gebrauch Grenzen.

\section{Klinische Wirksamkeit und therapeutische Anwendungsmöglichkeiten der NSAIDs}

Die große Zahl der akuten und chronischen Entzündungszustände am Bewegungsapparat, die klassische Indikationen für die NSAIDs beim Pferd darstellen, sind wohl bekannt und bedürfen keiner näheren Erläuterung. Dagegen gibt es einige neuere bemerkenswerte Anwendungsbereiche. Eine Gelegenheit, bei der solche NSAIDs herangezogen werden können, die als iv injizierbare Präparate im Handel sind, nämlich Phenylbutazon und Flunixin, ist die Koliktherapie. Sie erfreuen sich hier bei den Pferdepraktikern auch zunehmender Beliebtheit. Ebenso sind NSAIDs in steigendem Maß fester Bestandteil der zur Medikation herangezogenen Arzneimittel in postoperativen Behandlungsplänen. Der postoperativ herbeigeführte Erfolg liegt vor allem in den eikosanhemmenden, antientzündlichen und ödemhemmenden Eigenschaften dieser Stoffe begründet. Die Wundheilung verzögern sie, so hofft man, dabei nicht. Es fällt schwer, den Wert ihrer Anwendung objektiv mit Argumenten zu untermauern. Einige Chirurgen sind jedoch auf der Basis ihrer klinischen Erfahrung davon überzeugt.

\section{Phenylbutazon}

In den letzten Jahren geriet Phenylbutazon mehrfach in den Ruf, toxischer und weniger wirksam als die neuer eingeführten NSAIDs zu sein. Trotzdem ist es immer noch das am häufigsten angewandte Medikament seiner Art in der Pferdepraxis. Nach Ansicht von Snow (1981) liegt der Grund dafür zweifellos in der im Lauf von 30 Jahren erworbenen klinischen Erfahrung und den vergleichsweise niedrigeren Kosten der Phenylbutazon-Behandlung. Scott Dunn (1971) gab an, Phenylbutazon habe sich seiner Feststellung nach als besonders nützlich bei der Therapie von Arthritiden und osteoporotischen Zustandsbildern erwiesen.

Bei einem Modellfall von Lahmheit beim Pferd war die empfohlene iv Dosis Phenylbutazon ( $4,4 \mathrm{mg} / \mathrm{kg} \mathrm{KG}) 24 \mathrm{~h}$ lang wirksam. Der stärkste Effekt trat nach 8 bis $12 \mathrm{~h}$ auf (Houdesbell und Hennessey, 1977). Meßwerte aus dem Labor der Autoren bestätigen diese klinischen Befunde. In einem Modellfall von akuter Entzündung senkte Phenylbutazon die Prostaglandin- $\mathrm{E}_{2}$-Spiegel mindestens $24 \mathrm{~h}$ lang wesentlich (Higgins und Lees, 1983). Ausgehend von diesen beiden Untersuchungen, erscheint es wohl sinnvoll, die vorliegen-

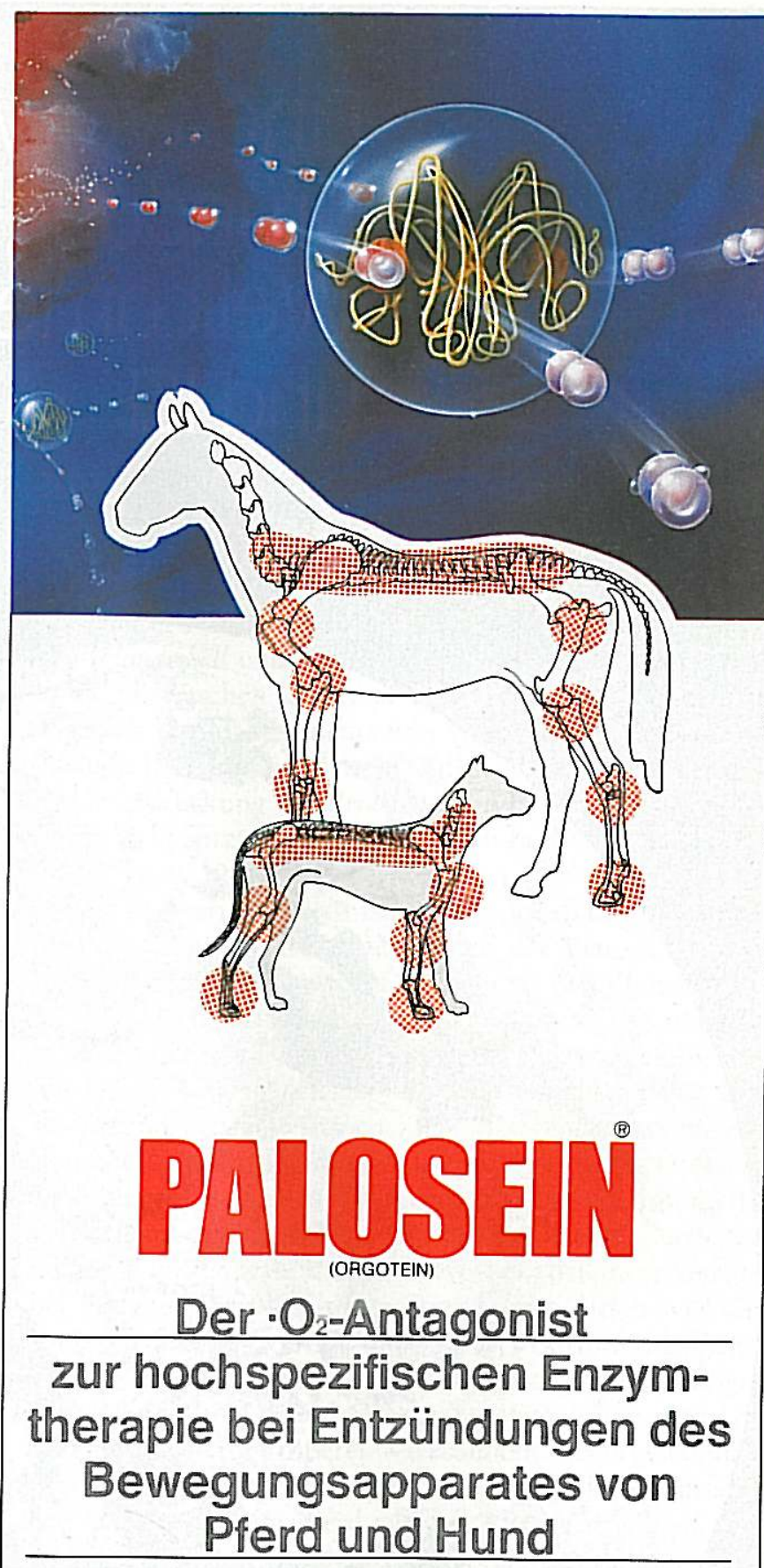

Zusammensetzung: Trockensubstanz und Lösungsmittel. 1 Injektionsflasche mit $15 \mathrm{mg}$ Trockensubstanz enthält $5 \mathrm{mg}$ Orgotein (aus Rinderleber mittleres MG 32600). 1 Ampulle mit 2 ml Lösungsmittel enthält $18 \mathrm{mg}$ Natriumchlorid. Anwendungsgebiete: Zur Behandlung akuter und chronischer Entzündungen, speziell: Pferd-Entzündungen und degenerative Veränderungen im Bereich des Bewegungsapparates, Hund - Bewegungsstörungen infolge entzündlicher und degenerativer Veränderungen im Bereich der Wirbelsäule. Gegenanzeigen: Bei der Behandlung von entzündlichen Vorgängen, die auf einer Infektion beruhen, ist zusätzlich zu PALOSEIN eine spezifische Behandlung der Infektion erforderlich. Im Falle von Rückfällen oder Wiedererkrankung sollten die Tiere auf Zeichen von Sensibilisierung beobachtet werden. Nebenwirkungen: Nicht bekannt. Wechselwirkungen mit anderen Mitteln: Nicht bekannt. Wartezeit: Keine. Die zubereitete Lösung sollte bei $4^{\circ} \mathrm{C}$ im Kühlschrank gelagert werden. Packungsgröße: Packung mit 6 Injektionsflaschen à $15 \mathrm{mg}$ Trockensubstanz + 6 Ampullen à $2 \mathrm{ml}$ Lösungsmittel.

Informieren Sie sich über das progressive Therapieprinzip bei CRLIMIEX

GRÜNTEX GMBH · PRODUKTE FÜR TIERGESUNDHEIT WERKSTRASSE 16 - TEL.: 02408-2041 5100 AACHEN 
den Dosierungsschemata für Phenylbutazon zu revidieren und sowohl für den oralen als auch für den parenteralen Applikationsweg Empfehlungen zu geben, die auf Verabreichung einmal täglich lauten. Für die anderen NSAIDs, die sogar eine kürzere Plasmahalbwertszeit besitzen, ist der Rat hinsichtlich einmaliger Anwendung pro Tag ohnehin schon erteilt. Die Plasmahalbwertszeit ist, so ist anzunehmen, nicht mehr unumstrittener Hauptbestimmungsfaktor für die Dosierungsintervalle der NSAIDs.

In Fällen von Kolik ist bei iv Injektion Phenylbutazon unter Umständen von Nutzen, dann aber hochdosiert $(15 \mathrm{mg} / \mathrm{kg} \mathrm{KG}$ als Initialdosis, gefolgt von Gaben mit 10 $\mathrm{mg} / \mathrm{kg}$ nach 6 bzw. $12 \mathrm{~h}$ ). In diesen Mengen hat Phenylbutazon bei Ponies mit akuter experimenteller Escherichiacoli-Endotoxämie verschiedene pathologische Veränderungen im Kreislauf und bei biochemischen Vorgängen verhindert oder niedergehalten (Burrowes, 1981). Allerdings verbesserte diese Behandlung nicht die eventuelle Überlebensrate.

\section{Naproxen}

In den vergangenen etwa 10 Jahren haben Forscher in den USA eine Reihe von Labormodellen für die Entzündungsvorgänge beim Pferd entwickelt, um besser die Wirksamkeit der NSAIDs untersuchen zu können. Einige dieser Modelle gerieten in die Kritik, sie seien ethisch fragwürdig, aber nichtsdestoweniger ließ sich mit ihrer Hilfe ein brauchbarer Zustand herstellen, der sich von den in diesem Bereich anzutreffenden klinischen Störungen nicht zu stark unterschied. Zum Beispiel entwickelten Killian et al. (1974) das induzierte Myositis-Modell beim Pferd. Sie injizierten Milchsäure in die Rückenmuskulatur und versuchten, damit ein "Tying up"-Syndrom zu erzeugen. An Hand dieses Modells ließ sich zeigen, daß Naproxen in klinischer Dosierung ( 2 bis $4 \mathrm{~g}$ pro Tier 7 Tage lang zweimal täglich) hochwirksam bei der Unterdrückung bestimmter klinischer Manifestationsformen der entzündlichen Reaktion ist. Dazu gehören Schrittverkürzung, Schmerz, Umfangsvermehrung und Lahmheit. Bei einem zweiten Versuchsschritt stellte man die Wirkungen von Naproxen und Phenylbutazon denjenigen einer Plazebo-Behandlung gegenüber. Hier zeigten beide im Vergleich zum Plazebo beachtliche entzündungshemmende Effekte (Jones und Hamm, 1978). Naproxen war unter diesen Versuchsbedingungen stärker wirksam als Phenylbutazon, dessen Wirkung anscheinend viel langsamer eintrat. Endgültige Schlußfolgerungen läßt dieser Befund nicht zu, da jedes Medikament nur in einer einzigen Dosierung zur Anwendung kam.

Weder bei dieser noch bei anderen Untersuchungen am Pferd hat man das Verhältnis von Dosis und Reaktion bestimmt. Es ließe sich entweder in prozentualer Hemmung der Zyklooxygenase oder in klinischer Wirksamkeit ausdrücken. Eine weiteren Einblick versprechende Vergleichsstudie wäre daher, die Dosis zu bestimmen, mit der ein vorgegebener Grad an Enzymhemmung, sagen wir 90 Prozent, zu erreichen ist, und dann die klinische Effizienz dieser Dosierung bei verschiedenen Arzneimitteln zu vergleichen. Unterdessen sei noch darauf hingewiesen, daß die Phenylbutazonmenge, die Jones und Hamm (1978) appli- zierten, größer als die heute empfohlene (Tabelle 2) war, während ihre Naproxendosis derjenigen entsprach, zu der auch zur Zeit geraten wird.

Tobin (1979b) berichtete über die klinische Wirksamkeit von Naproxen in klinischen Fällen von Lumbago. Er erzielte in 90 Prozent der Fälle ein gutes Ergebnis, wobei die Remissionszeit durchschnittlich 5 Tage betrug. Naproxen scheint besonders gut wirksam bei Entzündungszuständen des Bindegewebes zu sein.

\section{Flunixin}

Nach oraler oder iv Verabreichung tritt die Wirkung von Flunixin beim Pferd nach $2 \mathrm{~h}$ ein. Der stärkste Effekt ist zwischen der 2. und 16. Stunde festzustellen, und in abgeschwächter Form ist bis zu $30 \mathrm{~h}$ lang eine Wirkung vorhanden (Houdeshell und Hennessey, 1977). Diese klinischen Befunde überraschen in Anbetracht der kurzen Plasmahalbwertszeit des Medikaments von 1,6 h. Sie haben nun aber dadurch Bestätigung erfahren, daß sich eine 12 bis $24 \mathrm{~h}$ lange Unterdrückung der Prostaglandin- $\mathrm{E}_{2}$-Produktion durch Flunixin in entzündlichen Exsudaten nachweisen ließ (Lees und Higgins, 1984).

In einem experimentellen Lahmheitsmodell beim Pferd, geschaffen durch tiefes Punktbrennen der Beugesehnen im Fesselbereich, wurden drei Dosisgrößen von Flunixin drei verschiedenen Gaben von Phenylbutazon gegenübergestellt. Flunixin war dabei viermal so wirksam ( $\mathrm{mg}$ für $\mathrm{mg}$ ) wie Phenylbutazon (Houdeshell und Hennessey, 1977). Dieser Befund bedeutet jedoch nicht notwendigerweise, daß Flunixin klinisch effektiver ist. Ein Vergleich der Dosen eines jeden der beiden Medikamente, die zur Hemmung der Prostaglandinsynthese im entzündlichen Exsudat erforderlich sind, würde wahrscheinlich ergeben, daß diese Mengen bei Patienten mit klinischen Symptomen gleich wirksam sind. Solange das Verhältnis der zur Erzielung klinischer Effekte erforderlichen Dosen und dasjenige der jeweiligen Menge, bei deren Verabreichung Toxizität zu erwarten ist, gleich sind, ist mit größerer Wirksamkeit nichts gewonnen. Freilich ist der Sicherheitsspielraum von Flunixin hinsichtlich Dosierung beim Pferd zweifellos ein Vorteil.

Zusätzlich zu seinem Wert als Entzündungshemmer in einem großen Bereich von Störungen am Bewegungsapparat ist Flunixin besonders als Analgetikum bei der Kolikbehandlung empfohlen (Vernimb und Hennessey, 1977). Bei dieser Untersuchung zeigten zwei Drittel der behandelten Tiere eine sehr gute und rasche klinische Reaktion. Leider beschränkte sich die Beurteilung allein auf das klinische Bild, und keine Kontrolluntersuchungen erfolgten. Ungeachtet dessen war den Angaben der Forscher gemäß ihr Erfolg in Fällen von Gaskolik am größten und am kleinsten bei Patienten mit Ileus. Eine teilweise Besserung trat auch bei 4 von 6 Ponies mit experimenteller Kolik ein. Die Wirkungsweise des Flunixins bei diesen Patienten ist nicht geklärt. In Betracht kommt ein zentraler analgetischer Effekt zusammen mit einer lokalen Beeinflussung der EikosanProduktion im Darm. Eine weitere Anwendungsmöglichkeit von Flunixin besteht bei der Therapie des EndotoxinSchocks. In zwei Versuchsanordnungen rief Flunixin günstige Wirkungen bei anästhesierten Ponies hervor, die E.- 
coli-Endotoxin erhalten hatten. Die Laktat-Azidose milderte sich, der niedrige Blutdruck war nicht mehr so ausgeprägt und der Blutrückfluß aus den Venen verbessert. Im Magen-Darm-Trakt ließ sich eine starke Vasodilatation verhindern, nicht dagegen der Anstieg des Hämatokrits (Bottoms et al., 1981; Fessler et al., 1982). Bei einer Folgeuntersuchung, ausgelegt zur Nachahmung des Kolikverlaufs beim Pferd, verhinderte Flunixin einen Anstieg der Konzentration von Thromboxan und Prostazyklin in der Blutzirkulation, den das Endotoxin verursacht. Die Überlebensrate war verbessert; von 8 Ponies waren ohne Behandlung 6, unter der Flunixin-Therapie dagegen nur 2 gestorben (Templeton, Bottoms und Fessler, 1983).

Bei einem anderen Endotoxin-Experiment, diesmal an nichtnarkotisierten Ponies, verhütete die prophylaktische Flunixin-Behandlung verschiedene durch das Endotoxin ausgelöste Veränderungen, darunter Dyspnoe, Hypoxämie, Kolik und Laktat-Azidose (Moore, Garner, Shapland und Hatfield, 1981). Die Beobachtungen wiesen auf die wichtige Rolle der Eikosane beim Endotoxin-Schock hin. Gleichzeitig deuteten sie auf eine neue beachtenswerte Anwendungsmöglichkeit der NSAIDs hin. Allerdings ist die Behandlung eines bereits eingetretenen Schockzustandes mit Flunixin wahrscheinlich weniger erfolgreich als die Prophylaxe. Die schweren, lebensbedrohlichen Veränderungen in Fällen mit klinischen Schocksymptomen sind keinesfalls alle Produkten des Zyklooxygenase-Stoffwechsels zuzuschreiben. Bevor der volle Wert des Flunixins und verwandter Stoffe abschätzbar ist, muß man noch klinische Erfahrungen sammeln.

\section{Meclofensäure}

Meclofensäure ist nur als Granulat zum oralen Gebrauch im Handel. Durch ihre hohe Wirksamkeit sind lediglich kleinere Mengen ( $\mathrm{mg}$ für $\mathrm{mg}$ ) als von anderen NSAIDs erforderlich, Flunixin ausgenommen. Außerdem führt trotz einer Plasmahalbwertszeit von 2,5 h die einmalige Verabreichung pro Tag offenbar annehmbare klinische Effekte herbei. Über den Zeitraum, in dem das Arzneimittel die Produktion der im Zyklooxygenase-Stoffwechsel entstehenden Eikosane an entzündlich veränderten Stellen unterdrückt, existieren keine Angaben. Vermutlich gleicht es darin aber Phenylbutazon und Flunixin und wirkt $12 \mathrm{~h}$ oder länger. Die Zeitspanne bis zum Einsetzen der Wirkung von Meclofensäure scheint groß zu sein, nach der ersten Dosis dauert es 36 bis 96 h (Conner et al., 1973). Dies ist untypisch für NSAIDs beim Pferd, und der Grund dafür ist unbekannt. Eine langsame Absorption ist nicht verantwortlich, denn normalerweise ist innerhalb von 0,5 bis $4 \mathrm{~h}$ nach der Applikation die maximale Plasmakonzentration erreicht (Snow et al., 1981a). Was immer die Ursache sein mag, für die Klinik bedeutet diese lange Latenzzeit wohl, daß die klinische Effizienz in ihrem vollen Ausmaß erst nach zwei- bis viertägiger Anwendung abzusehen ist. Wiederholte tägliche Verabreichung über einen Zeitraum von 10 Tagen hinweg führt nicht zur Kumulation des Wirkstoffs im Plasma (P. Lees und W. E. Allen, unveröffentlichte Daten).
Die klinischen Indikationen entsprechen etwa den akuten und chronischen Entzündungsvorgängen, für die auch andere NSAIDs empfohlen sind. Der klinischen Erfahrung nach zu urteilen, scheint Meclofensäure jedoch besonders bei akuter und chronischer Rehe sowie bei Skeletterkrankungen gute Dienste zu leisten (Snow, 1981). Bei einer Untersuchung zeigten 76 Prozent der an Rehe erkrankten Pferde, 78 Prozent der Pferde mit Hufrollenveränderungen und 61 Prozent derer mit Osteoarthritis klinisch Besserung (Conner et al., 1973). In einem Doppel-Blindversuch wurden Phenylbutazon $(8,8 \mathrm{mg} / \mathrm{kg} \mathrm{KG}$ am ersten Tag, danach 7 Tage lang 4,4 mg/kg KG) und Meclofensäure (7 Tage lang $2,2 \mathrm{mg} / \mathrm{kg} \mathrm{KG}$ ) in ihrer Wirkung bei Pferden mit Hufrollenerkrankung oder Osteoarthritis verglichen. Bei 60 bzw. 36 Prozent der Pferde, die Meclofensäure bzw. Phenylbutazon erhalten hatten, kam es zu einer positiven klinischen Reaktion (G. A. Smith, persönliche Mitteilung).

\section{Ethische Gesichtspunkte bei der NSAID-Anwendung}

Hamm (1978) untersuchte den Gebrauch von Naproxen in der Applikation bei Jährlingen, die in die Auswahl fürs Rennen gekommen waren. Die Dosierung betrug $4 \mathrm{~g}$ zweimal täglich über den Zeitraum einer viermonatigen Trainingsphase und einer darauffolgenden viermonatigen Rennsaison hinweg. In der Gruppe der naproxenbehandelten Tiere traten laut Bericht weit weniger Trainingsunterbrechungen und weniger Erkrankungen des Bewegungsapparats auf. Dies kann kaum überraschen, und ganz ohne Zweifel ließen sich für die Anwendung anderer NSAIDs ähnliche Wirkungen nachweisen. Eine solche Prophylaxe ist aber laut Gesetz im UK oder vielen anderen Ländern nicht erlaubt.

Ebenso sollten auch einige Überlegungen der Frage gelten, in welchem Ausmaß ein solcher Arzneimittelgebrauch vom ethischen Standpunkt aus noch zu rechtfertigen ist, sowie den Konsequenzen für das Wohlergehen der Pferde auf längere Sicht. Die Ethik ist bei der NSAID-Verabreichung an Wettkampfpferde ein kontroverser Diskussionspunkt, auf den Snow (1983) vor kurzem näher eingegangen ist. Die Argumente für und gegen die Anwendung sollen hier nicht wiederholt werden. Es seien nur folgende Betrachtungen zum Problem der Festlegung einer Obergrenze für die noch akzeptable Plasmakonzentration von NSAIDs angestellt.

Die Bestimmung des Blutalkoholspiegels beim Menschen im Zusammenhang mit den gesetzlichen Vorschriften über Alkohol am Steuer hat eine unabdingbare soziale Funktion, und die Interpretation der Meßwerte ist einigermaßen geradlinig. Die Effekte von Alkohol auf das Zentralnervensystem sind direkte und laufen nicht über eine Aktivierung im Stoffwechsel. Der Grad der zentralnervösen Dämpfung steht in direkter Beziehung zur Alkoholkonzentration im Gehirn, und die Gehirnkonzentrationen gleichen sich mit ziemlich geringer zeitlicher Verschiebung an die Blutspiegel an. Mit anderen Worten, eine vorgegebene Blutkonzentration verursacht einen relativ gut vorherbestimmbaren Grad an Einschränkung der zentralnervösen Funktionen. Sicher bestehen individuelle Unterschiede, aber ihre Größe 
kann den Wert der interpretierten Meßdaten nicht mindern. In analoger Weise, so lautet die Argumentation, läßt sich aus der Kenntnis der Plasmakonzentration eines Wirkstoffs, die zur Erzielung eines vorgegebenen therapeutischen Effekts erforderlich ist, eine Obergrenze für NSAIDs bei Pferden festsetzen, die im Wettkampf stehen.

Nach diesem Schema geht die FEI bei Phenylbutazon vor. Es läßt zur Zeit eine Höchstkonzentration von $4 \mu \mathrm{g} / \mathrm{ml}$ Plasma zu. Wenn ausreichend Daten zur Pharmakokinetik und Toxikologie zur Verfügung stehen, werden die Bestimmungen sehr wahrscheinlich auf andere NSAIDs ausgedehnt. Die Anwendung und Interpretation dieser Daten ist jedoch alles andere als geradlinig. Erstens besteht nicht wie beim Blutalkoholspiegel eine einfache Beziehung zwischen der Plasmakonzentration und der Konzentration am Ort der Wirkung. Die Plasmakonzentration von Phenylbutazon $6 \mathrm{~h}$ nach iv Injektion und diejenige von Flunixin $3 \mathrm{~h}$ danach liegt über der im entzündlichen Exsudat. Nach $12 \mathrm{~h}$ (Phenylbutazon) bzw. $6 \mathrm{~h}$ (Flunixin) ist sie im Exsudat höher als im Plasma (Tabelle 4). Unter Berücksichtigung dieser Verkleinerung des Plasmakonzentration-Exsudatkonzentration-Verhältnisses mit fortschreitender Zeit kann man auf der Grundlage einer einzigen Blutprobe nicht sagen, ob die Konzentration an entzündlich veränderten Stellen zum Zeitpunkst der Probenentnahme über oder unter dem Plasmaspiegel liegt. Zweitens wandelt die Leber des Metabolit ebenfalls entzündungshemmende Eigenschaften besitzt, muß jede Beurteilung der Wirkungsstärke von Phenylbutazon auch dieses Stoffwechselprodukt in die Rechnung einbeziehen. Es tritt im akut entzündlichen Exsudat wie sein Vorläufer in nachweisbarer und nicht vorherbestimmbarer Menge auf (Tabelle 4).

Drittens haben wir kurz zuvor dargelegt, daß sowohl Phenylbutazon als auch Flunixin die Prostaglandin- $\mathrm{E}_{2}-\mathrm{Sp}$ iegel in beträchtlichem Maß niederhalten, was wahrscheinlich als guter Indikator für die anzunehmende Dauer ihrer entzündungshemmenden Wirkung heranziehbar ist. Sie tun dies nach iv Injektion einer einzigen therapeutischen Dosis 12 bis 24 h lang. Im Gegensatz dazu waren die PlasmaWirkstoffspiegel nach $24 \mathrm{~h}$ sehr niedrig, ein Zeichen für die kurze Halbwertszeit dieser Stoffe. Es ist daher denkbar, daß die Zyklooxygenase-Hemmung und vermutlich auch die entzündungshemmende Wirkung einige Zeit über den Abfall der Plasma-Wirkstoffkonzentration auf niedrige Werte hinaus anhalten. Die Messung der NSAID-Konzentration in einer einzelnen Blutprobe kann den angenommenen Effekt des Medikaments bei dem Tier nur näherungsweise und indirekt andeuten. Um unterhalb zulässiger Spiegel zu bleiben, handelt man am ehesten im Interesse des Pferdes, wenn man sie niedrig wählt.

Für die Pferdepraxis bieten wir an:

\section{Einreibungen zur Erzeugung einer örtlichen Hypere̊imic}

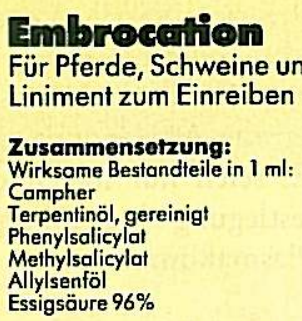

Anwendungsgebiete:

Verrenkungen, Verstauchungen, Quetschungen, Geschirr- und Satteldruck, Rheumatismus

\section{Wartezeit:}

Eßbares Gewebe

\section{Handelsform:}

Flasche mit $200 \mathrm{ml}$
Jodlysin ${ }^{\circledR}$

Für Tiere

Lösung zum Einreiben

Zusammensetzung:

Wirksame Bestandteile in $1 \mathrm{ml}$ :

Jod

Campher

Terpentinöl, gereinig

Ammoniaklösung konz.

Anwendungsgebiete:

Akute und chronische Entzündungs-

prozesse, insbesondere der Sehnen und Sehnenscheiden, Distorsionen.

Wartezeit:

Eßbares Gewebe

3 Tage

Handelsform:

Flasche mit $100 \mathrm{~m}$

Flasche mit 1 Liter

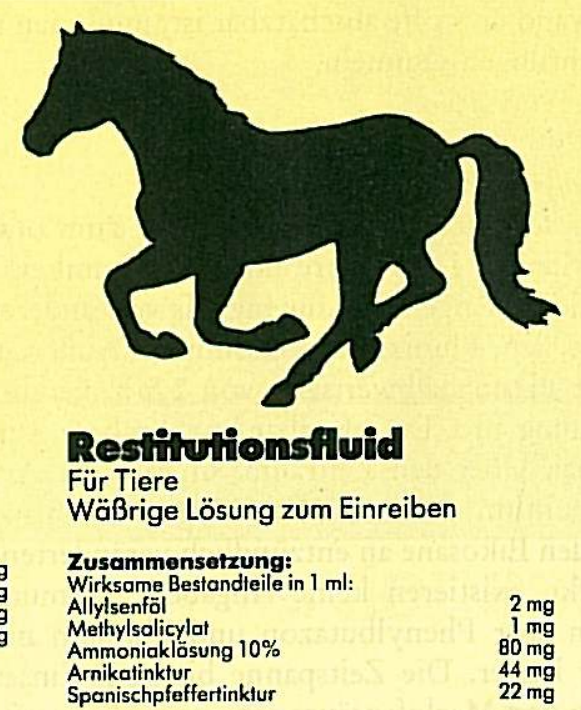

Anwendungsgebiete:

Lahmheiten, Sehnenentzündungen,

Verstauchungen, rheumatische

Erkrankungen

Wartezeit:

EBbares Gewebe

3 Tage

Handelsform

Flasche mit $250 \mathrm{ml}$

Flasche mit 1 Liter

\section{Wirtschaftsgenossenschaft deutscher Tierärzte eG, Dreyerstraße 8-12, 3000 Hannover 1, Tel. (05 11) 15143-46}




\section{Literatur}

Alexander, F. (1982): Effect of phenylbutazone on electrolyte metabolism in ponies. Vet. Rec. 110, 271-272.

Bottoms, G. D., Fessler, J. F., Roesel, O. F., Moore, A. B., and Frauenfelder, H. C. (1981): Endotoxin-induced hemodynamic changes in ponies: effects of flunixin meglumine. Am. J. vet. Res. 42, 1514-1518.

Burrows, G. E. (1981): Therapeutic effect of phenylbutazone on experimental acute Escherichia coli endotoxemia in ponies. Am. J. vet. Res. 42, 94-99.

Chay, S., Woods, W. E., Nugent, T., Blake, J. W., and Tobin, T. (1982): The pharmacology of non-steroidal anti-inflammatory drugs in the horse: flunixin meglumine (Banamine). Equine Pract. 4, 16-23.

Conner, G. H., Riley, W. F., Beck, C. C., and Coppock, R. W. (1973): Arquel (CI-1583) A new non-steroidal anti-inflammatory drug for horses. Proc. Am. Ass. equine Pract. 19, 81-90.

Davis, L. E., and Westfall, B. A. (1972): Species differences in biotransformation and excretion of salicylate. Am. J. vet. Res. 33, 1253-1262.

Davis, L. E., Westfall, B. A., and Short, C. R. (1973): Biotransformation and pharmacokinetics of salicylate in newborn animals. Am. J. vet. Res. 34, 1105-1108.

Dun, F. (1895): Veterinary Medicines, Their Actions and Uses, 9th edn. David Douglas, Edinburgh. 601-605.

Dunavant, M. L., and Murray, E. S. (1975): Clinical evidence of phenylbutazone-induced hypoplastic anemia. Proc. 1st Int. Symp. Equine Hematol. 353-355.

Fessler, J. F., Bottoms, G. D., Roesel, O. F., Moore, A. B., Frautenfelder, H. C., and Boon, G. D. (1982): Endotoxin-induced change in hemograms, plasma enzymes and blood chemical values in anesthetized ponies: effects of flunixin meglumine. Am. J. vet. Res. 43, 140-144.

Flower, R. J. (1974): Drugs which inhibit prostaglandin biosynthesis. Pharmacol. Rev. 26, 33-67.

Gabel, A. A., Tobin, T., Ray, R. S., and Maylin, G. A. (1977): Phenylbutazone in horses: a review. J. equine Med. Surg. 1, 221-225.

Gabriel, K. L., and Martin, J. E. (1962): Phenylbutazone: short-term versus long-term administration to thoroughbred and standardbred horses. J. Am. vet.med. Ass. 140, 337-341.

Gerring, E. L., Lees, P., and Taylor, J. B. (1981): Pharmacokinetics of phenylbutazone and its metabolites in the horse. Equine vet. J. 13, 152-157.

Gunson, D. E. (1983): Renal papillary necrosis in horses. J. Am. vet. Ass. 182, 263-266.

Gunson, D. E., and Soma, L. (1983): Renal papillary necrosis in horses after phenylbutazone and water deprivation. Vet. Pathol. 20, 603-610.

Hamm, D. (1978): Continuous administration of naproxen to the horse during training. J. equine Med. Surg. 2, 125-128.

Higgins, A. J., and Lees, $P$. (1983): Phenylbutazone inhibition of prostaglandin $E_{2}$ production in equine acute inflammatory exudate. Vet. Rec. 113, 622-623.

Higgins, A. J., and Lees, P. (1984): The acute inflammatory process, arachidonic acid metabolism and the mode of action of anti-inflammatory drugs. Equine vet. J. 16, 163-174.

Higgins, A. J., Lees, P., and Taylor, J. B. (1984): Influence of phenylbutazone on eicosanoid levels in equine acute inflammatory exudate. Cornell Vet. 74, 198-207.

Higgs, G. A., Flower, R. J., and Vane, J. R. (1979): A new approach to antiinflammatory drugs. Biochem. Pharmac. 28, 1959-1961.

Higgs, G. A., Palmer, R. M. J., Eakins, K. E., and Moncada, S. (1981): Arachidonic acid metabolism as a source of inflammatory mediators and its inhibition as a mechanism of action for anti-inflammatory drugs. Molec. Aspects Med. 40, 275-301.

Hopes, $R$. (1972): Uses and misuses of anti-inflammatory drugs in racehorses. Equine vet. J. 4, 66-68.

Houdeshell, J. W., and Hennessey, P. W. (1977): A new nonsteroidal anti-inflammatory analgesic for horses. J. equine Med. Surg. 1, 57-63.

Hunt, J. P., Haywood, P. E., and Moss, M. S. (1979): A gas chromatographic screening procedure for the detection of non-steroidal anti-inflammatory drugs in horse urine. Equine vet. J. 11, 259-263.

Janchen, E., and Levy, G. (1972): Inhibition of phenylbutazone elimination by its metabolite oxyphenbutazone. Proc. Soc. exp. Biol. Med. 141, 963-965.
Jeffcott, L. B., and Colles, C. M. (1977): Phenylbutazone and the horse - a review. Equine vet. J. 9, 105-110.

Jenny, E., Steinijans, V. W., and Seifert, P. (1979): Pharmacokinetic interaction of isopropylaminophenazone and phenylbutazone in the horse. J. vet. Pharmacol. Therap. 2, 101-108.

Johnstone, I. B. (1983): Comparative effects of phenylbutazone, naproxen and flunixin meglumine on equine platelet aggregation and platelet factor 3 availability in vitro. Can. J. comp. Med. 47, 172-179.

Jones, E. W., and Hamm, D. (1977): Steroidal and non-steroidal anti-inflammatory drugs for wounds and traumatic inflammation. $N$. Z. vet. J. 24, 317-319.

Jones, E. W., and Hamm, D. (1978): Comparative efficacy of phenylbutazone and naproxen in induced equine myositis. J. equine Med. Surg. 2 , 341-347.

Judson, D. G., and Barton, M. (1981): Effect of aspirin on haemostasis in the horse. Res. vet. Sci. 30, 241-242.

Kampmann, E., and Frey, H. (1966): Serum concentration of phenylbutazone in tests for anti-phlogistic activity and under clinical treatment. Nature 209, 579-582.

Killian, J. G., Jones, E. W., Hamm, D., Riley, W. F., and Averkin, E. (1974): The efficacy of Equiproxen (naproxen) in a unique equine myositis model. Proc. 20th Ann. Conv. Am. Ass. equine Pract. 201-215.

Lees, P., and Higgins, A. J. (1984): Flunixin inhibits prostaglandin production in equine inflammation. Res. vet. Sci. 37, 347-349.

Lees, P., Maitho, T. E., Millar, J. D., and Taylor, J. B. (1982): Pharmacokinetics of phenylbutazone in Welsh Mountain ponies. Pharmacologie, Toxicologie Vétérinaires, Inra Publ. Paris. 159-160.

Lees, P., Creed, R. F. S., Gerring, E. E. L., Gould, P. W., Humphreys, D. J., Maitho, T. E., Michell, A. R., and Taylor, J. B. (1983a): Biochemical and haematological effects of phenylbutazone in horses. Equine vet. J. 15, 158-167.

Lees, P., Maitho, T. E., Millar, J. D., and Taylor, J. B. (1983b): Pharmacokinetics of phenylbutazone in Welsh Mountain ponies. AVCPT Proceedings 7, 32-37.

Lebmann, W. von, Wintzer, H.J., and Frey, H.H. (1981): Kinetik einiger analgetisch-antiinflammatorischer Arzneimittel in Serum und Synovia beim Pferd. Deutsche Tierärzt. Wochenschrift 88, 218-220.

Mackay, R. J., French, T. W., Nguyen, H. T., and Maybew, I. G. (1983): Effects of large doses of phenylbutazone administration to horses. Am. J. vet. Res. 44, 774-780.

Maitho, T. E., Lees, P., and Taylor, J. B. (1985): Absorption and pharmacokinetics of phenylbutazone in Welsh Mountain ponies. J. Vet. Pharmacol. Ther. (in press)

Maylin, G. A. (1974): Disposition of phenylbutazone in the horse. Proc. 20th Conv. Ass. Am. equine Pract. 243-249.

Meschter, C. L., Maylin, G. A., and Krook, L. (1984): Vascular pathology in phenylbutazone intoxicated horses. Cornell Vet. 74, 282-297.

Moore, J. N., Gamer, H. E., Shapland, J. E., and Hatfield, D. G. (1981): Prevention of endotoxin-induced arterial hypoxaemia and lactic acidosis with flunixin meglumine in the conscious pony. Equine vet. J. 13, 95-98.

Moos, M. S. (1972): Uses and misuses of antiinflammatory drugs in racehorses - II. Equine vet. J. 4, 63-72.

Phillipps, M. W., Salyer, G., and Ray, R. S. (1980): Equine metabolism and pharmacokinetics of indomethacin. Equine Pract. 2, 45-49.

Piperno, E., Ellis, D. J., Getty, S. M., and Brody, T. M. (1968): Plasma and urine levels of phenylbutazone in the horse. J. Am. vet. med. Ass. 153, 195-198.

Read, W. K. (1983): Renal medullary crest necrosis associated with phenylbutazone therapy in the horse. Vet. Pathol. 20, 662-669.

Roberts, M. C. (1981): Suspected phenylbutazone toxicity in an adult stockhorse. Aust. vet. Pract. 11, 112-114.

Roberts, M. C. (1982): Adverse effects of indomethacin in the horse. J. vet. Pharmacol. Therap. 5, 83-86.

Rose, R. J., Kobnke, J. R., and Baggot, J. D. (1982): Bioavailability of phenylbutazone preparations in the horse. Equine vet. J. 14, 234-237.

Ruckebusch, Y., and Toutain, P. L. (1983): Nonsteroidal anti-inflammatory agents: species differences in pharmacodynamics. Vet. res. Comm. 7 , 359-368.

Sams, R. (1979): Pharmacokinetic and metabolic considerations as they apply to clinical pharmacology. In: Equine Pharmacology. Eds J. D. Powers and T. E. Powers. American Association of Equine Practitioners, Colorado. 17-23. 
Scott Dunn, $P$. (1971): A clinician's views on the use and misuse of phenylbutazone. Equine vet. J. 4, 63-65.

Snow, D. H. (1981): Non-steroidal anti-inflammatory agents in the horse. Vet. Rec. Suppl. In Pract. 3, 24-31.

Snow, D. H. (1983): Anti-inflammatory agents. In: Pharmacological Basis of Large Animal Medicine. Eds J. A. Bogan, P. Lees and A. T. Yoxall, Blackwell Scientific Publications, Oxford. 391-427.

Snow, D. H., Bogan, J. A., Douglas, T. A., and Thompson, H. (1979): Phenylbutazone toxicity in ponies. Vet. Rec. 105, 26-30.

Snow, D. H., Baxter, P., and Whiting, B. (1981a): The pharmacokinetics of meclofenamic acid in the horse. J. vet. Pharmacol. Therap. 4, 147-156.

Snow, D. H., Douglas, T. A., Thompson, H., Parkins, J. J., and Holmes, P. H. (1981b): Phenylbutazone toxicosis in Equidae: a biochemical and pathophysiologic study. Am. J. vet. Res. 42, 1754-1759.

Snow, D. H., and Douglas, T. A. (1983): Studies on a new paste preparation of phenylbutazone. Vet. Rec. 112, 602-607.

Snow, D. H., Douglas, T. A., Thompson, H., Holmes, P. H., and Parkins, $J$ J. (1983): Effect of non-steroidal anti-inflammatory agents on plasma protein concentration of ponies. Vet. Res. Comm. 7, 205-206.

Stone, E. (1763): An account of the success of the bark of the willow in the cure of agues. Phil. Trans. R. Soc. 53, 195.

Sullivan, M., and Snow, D. H. (1982): Factors affecting absorption of nonsteroidal anti-inflammatory agents in the horse. Vet. Rec. 110, 554-558.

Taylor, J. B., Walland, A., Lees, P., Gerring, E. L., Maitho, T. E., and Millar, J. D. (1983): Biochemical and haematological effects of a revised dosage schedule of phenylbutazone in horses. Vet. Rec. 112, 599-602.

Templeton, C. B., Bottoms, G. D., and Fessler, J. F. (1983): Effects of flunixin meglumine on hemodynamics, hematology, prostaglandins and survival in ponies during chronic exposure to endotoxin. Fed. Proc. 42, 323.

Tobin, T. (1979a): Pharmacology review: the nonsteroidal anti-inflammatory drugs. I. Phenylbutazone J. equine Med. Surg. 6, 253-258.

Tobin, T. (1979b): Pharmacology review: the non-steroidal anti-inflammatory drugs II. Equiproxen, meclofenamic acid, flunixin and others. J. equine Med. Surg. 6, 298-302.

Für die Unterstützung des Horserace Betting Levy Board (Project Grant 334) möchten wir dankbar unsere Anerkennung zum Ausdruck bringen. Wir sind zu Dank verpflichtet Dr. Gerald Higgs von den Wellcome Research Laboratories für seine hilfreichen Beiträge, Christine Kent für das Schreiben des Manuskripts und Leslie Dean für die Gestaltung der Abbildungen. Sehr dankbar sind wir für die Unterstützung der Hersteller von NSAID-Präparaten. Sie haben uns bisher unveröffentlichte Information zu ihren Produkten zur Verfügung gestellt.
Tobin, T., Blake, J. W., and Valentine, R. (1977): Drug interactions in the horse: effects of chloramphenicol, quinidine and oxyphenbutazone on phenylbutazone metabolism. Am. J. vet. Res. 38, 123-127.

Traub, J. L., Gallina, A. M., Grant, B. D., Reed, S. M., Gavin, P. R., and Paulsen, L. M. (1983 a): Phenylbutazone toxicosis in the foal. Am. J. vet. Res. 44, 1410-1418.

Traub, J. L., Paulsen, L. M., and Reed, S. M. (1983 b): The uses of phenylbutazone in the horse. Comp. Cont. Ed. pract. Vet. 5, 320-326.

Trillo, M. A., Soto, G., and Gunson, D. E. (1984): Flunixin toxicity in a pony. Equine Pract. 6, 21-29.

Trujillo, O., Rios, A., Maldonado, R., and Rudolph, W. (1981): Effect of oral administration of acetylsalicylic acid on haemostatis in the horse. Equine vet J. 13, 205-206.

Vane, J. R. (1973): Prostaglandins and aspirinlike drugs. In: Cellular Mechanismus, Vol. 5. Proceedings of the Fifth International Congress in Pharmacology. Eds R. A. Maxwell and G. H. Acheson. Karger, Basel. 353-378.

Vernimb, G. D., and Hennessy, P. W. (1977): Clinical studies on flunixin meglumine in the treatment of equine colic. J. equine Med. Surg. 1, 111-116.

Vogel, C. J. (1982): From firing to phenylbutazone in equine practice. Vet. Rec. 110, 523-525.

Wanner, F., Rollinghoff, W., Gerber, H., and Preisig, $R$. (1979): A preliminary report: demethylation, hydroxylation and acetylation in the horse. Side-effects of repeated phenylbutazone medication. In: Third Int. Symp. equine Med. Control. Eds T. Tobin, J. W. Blake and W. E. Woods. Lexington, Kentucky.

Webbon, P. M., and Woolliscroft, G. J. (1984): Cautious use of flunixin advocated. Vet. Rec. 115, 45.

Whittle, B. J. R., Higgs, G. A., Eakins, K. E., Moncada, S., and Vane, J. R. (1980): Selective inhibition of prostaglandin production in inflammatory exudates and gastric mucosa. Nature 284, 271-273.

Wilhelmi, G., and Mendasse-Gdynia, R. (1972): Gastric mucosal damage induced by non-steroid anti-inflammatory agents in rats of different ages. Pharmacology 8, 321-328.

Peter Lees

Department of Physiology

Royal Veterinary College

North Mymms, Hatfield, Hertfordshire AL9 7TA

United Kingdom.

Erschienen in Equine Veterinary Journal (1985) 17 (2), 83-96. Übersetzt und veröffentlicht mit freundlicher Genehmigung der British Equine Veterinary Association. 